\title{
Self-assembly of Sodium Bis(2-ethylhexyl) Sulfosuccinate in Aqueous Solutions: Modulation of Micelle Structure and Interactions by Cyclodextrins Investigated by Small-Angle Neutron Scattering
}

Ankitkumar I. Fajalia, Eleftheria Antoniou, Paschalis Alexandridis, Marina Tsianou*

Department of Chemical and Biological Engineering

University at Buffalo, The State University of New York (SUNY)

Buffalo, New York 14260-4200, USA

*Corresponding author:mtsianou@buffalo.edu 


\section{Abstract}

The modulating effects of $\alpha$-cyclodextrin ( $\alpha$-CD) and hydroxypropyl $\beta$-cyclodextrin (HP- $\beta$-CD) on micelles formed in water by the double tail surfactant sodium bis(2-ethylhexyl) sulfosuccinate (also known as dioctyl sulfosuccinate (DOSS) or Aerosol-OT (AOT)) have been investigated using small angle neutron scattering (SANS). SANS data were fitted to the core-shell oblate ellipsoid form factor and Hansen-Hayter-based structure factor in order to quantify the effect of cyclodextrins on the micelle structure and inter-micelle interactions. The results suggested that addition of cyclodextrins caused a sharp decrease in surfactant aggregation numbers, micelle dimensions and micelle charge. The micelle shape changed from elongated oblate to sphere upon addition of cyclodextrin at stoichiometric ratio of 1:1 CD:AOT for HP- $\beta$-CD and 2:1 for $\alpha-C D$. HP- $\beta-C D$ was found more effective than $\alpha-C D$ in influencing the micelle properties. For example, the decrease in surfactant aggregation number upon addition of $1 \mathrm{mM} \mathrm{CD}$ at constant total surfactant concentration was about double for HP- $\beta$-CD compared to $\alpha-C D$. The results are consistent with the localization of small amount of cyclodextrins in the micelle, while the remainder stays in the solution pseudophase. The present study offers an improved understanding on the modulating effects of CDs on micelle structure and enables better design of emulsifiers or dispersants in formulations suitable for controlled release of actives.

Keywords: sodium dioctyl sulfosuccinate, DOSS, Aerosol-OT, AOT, surfactant, micelle, cyclodextrin, small-angle neutron scattering, SANS, formulation. 


\section{Introduction}

Sodium bis(2-ethylhexyl) sulfosuccinate, also known as dioctyl sulfosuccinate (DOSS) or Aerosol-OT (AOT), is an anionic double-tail surfactant with relatively low solubility in water but high solubility in oil. ${ }^{1-2}$ Because of its remarkable interfacial properties, AOT has been widely used as stabilizer for microemulsions without the need of co-surfactant. ${ }^{3-7}$ Due to its relatively low toxicity and versatility, AOT finds applications as petroleum dispersant, ${ }^{8-10}$ as adjuvant or wetting agent in pesticide formulations, ${ }^{11}$ and as drug carrier in pharmaceutical formulations. ${ }^{12}$ AOT associates in water to form micelles at low concentrations, and lyotropic mesophases, e.g., hexagonal or lamellar, at higher concentrations. ${ }^{2,13-15}$ The critical micelle concentration (CMC) of AOT in water has been obtained from surface tension measurements to be $2.6 \mathrm{mM}$ (1.18 $\left.\mathrm{wt}_{\mathrm{t}} \%\right) .{ }^{15}$ As the surfactant concentration increases above the CMC, but below $29 \%$, AOT micelles grow from spherical to ellipsoid. ${ }^{2}$

The self-assembly of surfactants in water is modulated by the presence of co-solutes. ${ }^{16-18}$ In particular, the addition of cyclodextrins may affect the surfactant association because of their binding interactions with surfactant hydrophobic tails to form inclusion complexes. ${ }^{19}$ Cyclodextrins (CDs) are toroid shape supramolecules composed of $\alpha$-D-glucopyranoside units that form a macrocycle ring. ${ }^{20}$ Native cyclodextrins and their derivatives find a number of applications in pharmaceutical, cosmetic, and food industries for encapsulation of hydrophobic or volatile actives in the formulations, in analytical chemistry as separating agents, and in catalysis. $^{20-26}$ The exterior of the cyclodextrin molecules is hydrophilic while the central cavity offers a hydrophobic environment which is responsible for the binding of CD with surfactants.

Depending on the size of the central cavity and possible chemical modification, cyclodextrins form mainly $1: 1,2: 1$ or $1: 2 \mathrm{CD}$ :surfactant inclusion complexes with surfactants. ${ }^{19},{ }^{27-33}$ Specifically, the inclusion complexation of AOT with $\alpha$-cyclodextrin (6 member glucose ring), $\beta$-cyclodextrin ( 7 member glucose ring) and $\gamma$-cyclodextrin $(8$ member glucose ring) was examined using isothermal titration calorimetry ${ }^{34}$ to suggest that AOT forms primarily 1:1 $\mathrm{CD}$ :AOT complexes with $\alpha-, \beta$ - or $\gamma$-CD. The binding constant values of $170,180,490 \mathrm{M}^{-1}$ for 1:1 CD:AOT inclusion complexes of $\alpha-C D, \beta-C D$, and $\gamma-C D$, respectively, ${ }^{34}$ are lower compared 
to binding constants for 1:1 CD:surfactant complexation with the single-tail anionic surfactant sodium dodecyl sulfate (SDS) $\left(21000 \mathrm{M}^{-1} \text { for } 1: 1 \alpha \text {-CD:SDS or } \beta \text {-CD:SDS complexation }\right)^{30}$, which can be attributed to lower hydrophobic interactions between the two hydrophobic, branched tails of AOT and the CD cavity compared to the single-tail interactions of SDS with the CD cavity. ${ }^{34}$ Similar observations on the binding constants of $\beta$-cyclodextrin with AOT and other surfactants using conductivity measurements have been reported by Palepu et al. ${ }^{35}$

To the best of our knowledge, there are only two published studies on the complexation of cyclodextrins with AOT in aqueous solutions at AOT concentrations below the CMC. ${ }^{34-35}$ The effects of CD-AOT binding in water at AOT concentration higher than CMC have not been explored yet. Several questions need to be addressed: How does cyclodextrin binding affect the micelle size, shape and interactions? How does the cyclodextrin type affect the AOT micelles? Answer to these questions can be obtained by probing AOT-cyclodextrin solutions using a structural characterization technique such as small-angle neutron scattering (SANS). In a recent study, we have utilized SANS to investigate CD effects on micelle formation and structure in aqueous SDS solutions. SANS has been previously used for the characterization of aqueous solutions of AOT, either in the absence or in the presence of salt, ${ }^{2,13}$ however, SANS has never been used for studying AOT micelles in the presence of cyclodextrins.

The major objective of this work is to investigate using SANS how the micelle properties and inter-micelle interactions of the representative double-tail surfactant AOT are affected by cyclodextrins in aqueous solutions. We first consider AOT-water solutions as a reference system. We then examine AOT-cyclodextrin-water solutions with two different types of cyclodextrins, $\alpha$ cyclodextrin ( $\alpha-C D)$ and hydroxypropyl $\beta$-cyclodextrin (HP- $\beta-C D$ ), and compare their effectiveness. To the best of our knowledge, this is the first investigation of the AOTcyclodextrin-water system above the CMC, and one of only three published studies on AOT-CD aqueous solutions. 


\section{Materials and Methods}

\section{Materials}

Sodium bis(2-ethylhexyl) sulfosuccinate was purchased from Sigma (St. Louis, MO). $\alpha$ cyclodextrin and hydroxypropyl $\beta$-cyclodextrin were purchased from Aldrich (St. Louis, MO). Hydroxypropyl- $\beta-C D$ has been used in the present study due to its higher aqueous solubility compared to $\beta$-CD. The degree of substitution of hydroxypropyl groups for $\beta$-cyclodextrin was specified as 4.3 by the supplier. Heavy water $\left(\mathrm{D}_{2} \mathrm{O}\right)$ was purchased from Cambridge Isotope Laboratories (Tewksbury, MA). All chemicals were used as received.

Stock solutions of surfactant and of $\mathrm{CD}$ in $\mathrm{D}_{2} \mathrm{O}$ were first prepared, and then mixed in appropriate ratios with each other and with $\mathrm{D}_{2} \mathrm{O}$ to obtain the desired final concentration. Adequate time for equilibration was allowed. The concentration of AOT varied from $2 \mathrm{mM}$ to $22.5 \mathrm{mM}$ to cover a wide range of surfactant concentrations above the CMC but below the surfactant concentration where lyotropic liquid crystal phases form. ${ }^{2}$ The concentration of $\alpha-C D$ varied from 0 to $40 \mathrm{mM}$, while the concentration of HP- $\beta-C D$ varied from 0 to $20 \mathrm{mM}$, above which all micelles were disassembled for an AOT concentration of $12 \mathrm{mM}$.

\section{SANS measurements and data reduction}

SANS measurements were performed on the $30 \mathrm{~m}$ spectrophotometer (NG3) at the National Institute of Standards and Technology (NIST) in Gaithersburg, MD. Neutrons with $6 \AA$ wavelength and wavelength spread $\Delta \lambda / \lambda=14.3 \%$ were focused on the sample kept in a $4 \mathrm{~mm}$ quartz cell at $25 \pm 0.1^{\circ} \mathrm{C}$. Sample-to-detector distance $=2 \mathrm{~m}$ for all samples, covering the wave vector $(q)$ range $0.01 \AA^{-1}<q<0.3 \AA^{-1}$. The beam time was 180 to $4800 \mathrm{~s}$ for each measurement. Scattering data were corrected for scattering originating from background, empty cell and detector sensitivity. Absolute scale intensity was obtained by normalizing the scattered intensity with a well characterized polystyrene/deuterated polystyrene standard. ${ }^{32,}{ }^{36}$ Absolute intensity data were then corrected for incoherent solvent scattering and analyzed using different models available in NIST-supplied analysis software. Reduced SANS data were fitted to appropriate models for form and structure factors (see next section) using non-linear least square method 
with Levenberg-Marquardt algorithm to obtain physically appropriate values of the fitting parameters. The uncertainties in the fitting parameters are specified here as one standard deviation in the fitting coefficient and are calculated from the fitting function of IGOR PRO. ${ }^{31}$

\section{SANS data analysis: micelle structure and interactions}

The core-shell oblate ellipsoid form factor ${ }^{2,13}$ and the Hansen-Hayter structure factor ${ }^{37-38}$ are used in our study as the most appropriate in describing the scattering behavior of sodium bis(2ethylhexyl) sulfosuccinate micelles in aqueous solutions.

The overall scattering intensity $I(q)$ is given by equation (1):

$$
I_{\text {micelle }}(q)=A \cdot \phi \cdot P(q) \cdot S(q)+B_{\text {inc }}
$$

The form factor $P(q)$ represents the shape and structure of a micelle (Figure 2), while the structure factor $S(q)$ represents the inter-micelle interactions and overall arrangement of micelles in the solution. $\phi$ is the volume fraction of the micelles, which in turn depends on the overall surfactant concentration. The parameters $A$ and $B_{i n c}$ are introduced to take into account additional contributions associated with absolute scaling and incoherent noise, respectively. ${ }^{32}$

$P(q)$ was calculated using equation (2) and equation (3). ${ }^{32}$

$$
\begin{aligned}
& P(q)=\frac{1}{V_{\text {TOT }}} \int_{0}^{1}|F(q, a, b, \delta, \beta)|^{2} d \beta \\
& F(q, a, b, \delta, \beta)=V_{C}\left(\rho_{C}-\rho_{S h}\right) \frac{3 j_{1}\left(u_{C}\right)}{u_{C}}+V_{T O T}\left(\rho_{S h}-\rho_{S}\right) \frac{3 j_{1}\left(u_{T}\right)}{u_{T}}
\end{aligned}
$$

The first term in equation (3) represents scattering due to the core region of the micelle with core volume $V_{C}=\frac{4 \pi}{3} a^{2} b$, where $a$ represents the major core radius and $b$ represents the minor core radius. The second term in equation (3) signifies scattering due to the micelle shell region (with constant shell thickness $\delta$ as shown in Figure 2) of a micelle with volume $V_{\text {TOT }}=\frac{4 \pi}{3}(a+\delta)^{2}(b+\delta) \cdot j_{1}(u)$ is the first order spherical Bessel function of first kind 
expressed by $j_{1}\left(u_{i}\right)=\left(\sin u_{i}-u_{i} \cos u_{i}\right) / u_{i}^{2}$ where $u_{i}=q\left[a_{i}^{2} \beta^{2}+b_{i}^{2}\left(1-\beta^{2}\right)\right]^{1 / 2} \cdot \rho_{C}, \rho_{S h}$ and $\rho_{S}$ are the scattering length densities of the micelle core, micelle shell, and solvent, respectively. ${ }^{32}$ $\beta=\cos \mu$, where $\mu$ is the angle at which the major axis inclined to the scattering vector $q$. The use of monodisperse micelle form factor described here is appropriate for the higher-than-CMC surfactant concentrations that we use, where micelle-micelle interactions are prominent, and the effects of polydispersity on intra- or inter-particle structure factor are less pronounced. ${ }^{2,39}$

The structure factor $S(q)$ was calculated using the screened coulomb potential, with mean spherical approximation ${ }^{38}$ and rescaling corrections for low volume fractions, ${ }^{37}$ given the micelle volume fraction, charge on a micelle, and ionic strength of the solution. Micelles are approximated as charged spheres of diameter $\sigma$ interacting through ion-impenetrable dimensionless screened columbic potential ${ }^{40}$ and the solvent is considered as a continuum charge screening medium with inverse Debye length $\kappa$ as described by the Debye-Huckle theory. Since $\kappa \sigma<1$ (where $\kappa$ is the inverse Debye length and $\sigma$ is the effective diameter of the micelle) and the contact potential $\gamma \exp (-\psi) \square 1 \mathrm{mV}$, application of ion-impenetrable (hard-core) potential based structure factor is valid in our study. ${ }^{40}$ The effect of non-spherical shape on the structure factor can be neglected, i.e., the apparent structure factor $S^{\prime}(q)=S(q)$ with $\beta^{\prime}(q)=1$ where $S^{\prime}(q)=1+\beta^{\prime}(q)[S(q)-1]$ and $\beta^{\prime}(q)=\left\langle F(q, a, b, \delta, \beta)^{2}\right\rangle /\langle F(q, a, b, \delta, \beta)\rangle^{2}$, because the axis ratio of the micelles remains below 2 and $q_{\max } a$ remains below 1 ( $a \leq 17 \AA$ at $q_{\max } \leq 0.05 \AA^{-1}$ shown in Table 3 and Figure 3). ${ }^{41-42}$

The surfactant aggregation number $\eta$ (number of surfactant molecules per micelle), volume fraction of the micelles $\phi$, charge on a micelle $Z$, and cyclodextrin aggregation number $k$ (number of cyclodextrin molecules per micelle) were considered as the major fitting parameters to model scattering from the micelles. The micelle core radius $b$ was kept fixed at $b=12.57 \AA$ considering for fully stretched alkyl chain. ${ }^{2}$ In our previous study where we analyzed contrast variation SANS experiments, we established the localization of some cyclodextrin in the sodium dodecyl sulfate (SDS) micelles. ${ }^{32}$ AOT micelles are comparable in size with SDS micelles at the conditions considered in the present study, and hence are geometrically capable to accommodate a few cyclodextrin molecules as has been the case for SDS micelles. In order to assess the 
possible localization of CDs in AOT micellar solutions, the present SANS data were fitted using two different scenarios pertaining to the presence of CDs either all in solution or some in the micelle core and the remainder in solution. The fits to the SANS data when considering all CD to be in solution and none to partition in the micelles were found to be consistently poor compared to the scenario considering localization of a small fraction of CDs in the micelle. Accordingly, we consider in the present study a fraction of CDs to be localized in the AOT micelle core.

The micelle core volume $V_{C}\left(\AA^{3}\right)$ was calculated given the aggregation numbers for surfactant $\eta$ and cyclodextrin $k$ using $V_{C}=\eta V t_{A O T}+k V_{C D}$, where $V t_{A O T}$ and $V_{C D}$ represent the volume of hydrocarbon chains of the surfactant monomers and cyclodextrin molecules, respectively. Table 1 displays values of the above mentioned molecular parameters used for SANS data fittings. The tail volume of AOT was $V t_{A O T}=545.55 \AA^{-3}$ (calculated using the reported overall surfactant monomer volume of $611 \AA^{-3}$ and dry volume of the head group $65.45 \AA^{-3}$ ). ${ }^{2}$ The volume of a $\alpha$ CD or HP- $\beta$-CD molecule was $V_{C D}=795.5 \AA^{-3}$ or $V_{C D}=919.6 \AA^{-3}$, respectively (calculated from the CD dimensions given in Figure 1). The micelle major core radius $a$ was determined from the core volume using $a=\sqrt{3 V_{C} / 4 \pi b}$ from which the major-to-minor radius axis ratio $\varepsilon$ was determined. On the occasion where the value of ratio $\varepsilon$ was found to be $\varepsilon<1$, the data were fitted using core-shell sphere model with an equivalent spherical radius calculated using the same core volume $V_{C}$ determined before. In particular, at high $\alpha$-CD concentration (above 18 $\mathrm{mM}), \varepsilon$ drops below 1 if we use the ellipsoidal model with fixed $b=12.57 \AA$. We invoked the core-shell sphere model instead, allowing values of the core radius $b$ to be smaller (by $\sim 3 \%$ ) than $12.57 \AA$ during fittings at higher $\alpha$-CD concentrations. The total volume of a micelle was calculated using the core volume $V_{C}$ and the shell volume $V_{\text {Shell }}$ considering volume contributions from head groups, counterions and water molecules present in the shell using $V_{\text {Tot }}=\eta\left[V_{\mathrm{OSO}_{2}-}+(1-\alpha) V_{\mathrm{Na+}}+N_{\mathrm{H}_{D_{2} \mathrm{O}}}\right]+V_{\mathrm{C}}$, where $\alpha$ is the fractional charge on a micelle defined as $Z / \eta \cdot N_{H}$ is the hydration number (number of water molecules associated per surfactant molecule) of the surfactant head group, and was fixed at $N_{H}=21.5$ as reported by Sheu et al. ${ }^{2}$ $V_{\mathrm{OSO}_{2}-}, V_{\mathrm{Na}+}$, and $V_{\mathrm{D}_{2} \mathrm{O}}$ are the volume of head groups, counterions, and deuterated water 
molecule, respectively. The shell thickness $\delta$ was calculated iteratively from the total micelle volume and the micelle core volume by solving the equation $4 \pi(a+\delta)(b+\delta)^{2} / 3=V_{\text {TOT }}$.

Scattering length densities of the micelle core $\rho_{C}$ and shell $\rho_{S h}$ were calculated by addition of scattering length contributions of the groups/atoms present there (given in Table 1) and the molar volumes of the micelle core $V_{C}$ and the shell $V_{\text {Shell }}$ respectively. The scattering length density of solvent $\rho_{S}$ was calculated using equation (4) to include scattering contributions from the hydrogeneous cyclodextrin and surfactant molecules present in the deuterated water.

$$
\rho_{S}=\left[\frac{\rho_{D_{2} O}^{\text {mass }} \times 10^{6} b_{D_{2} O}}{M W_{D_{2} O}}+C_{C M C} b_{A O T}+(1-\Gamma) C_{C D} b_{C D}\right] N_{A v} \times 10^{-30}
$$

$\Gamma$ in equation (4) represents the fraction of cyclodextrin molecules that localize in the micelles which was calculated using the aggregation number of cyclodextrin $k$ and the number density of micelles $N_{P}$ by $\Gamma=N_{P} k /\left(C_{C D} N_{A v} \times 10^{-30}\right)$, where $N_{P}$ was calculated using $N_{P}=10^{-30}\left(C_{A O T}-C_{C M C}\right) N_{A V} / \eta$ given the surfactant concentration $C_{A O T}$. The volume fraction of the micelles $\phi$ was calculated using $\phi=N_{P} V_{T O T}$. The equivalent spherical diameter $\sigma_{0}$ of a micelle was calculated from $\sigma_{0}=2\left(\sqrt[3]{(a+\delta)^{2}(b+\delta)}\right)$.

The form factor, structure factor and overall scattering intensities were calculated using all the fitting parameters, fixed parameters, micelle dimensions and the scattering length densities utilizing NIST supplied routines. ${ }^{32}$ A similar fitting procedure has been used by Sheu et al. ${ }^{2}$ except that we have an additional fitting parameter, cyclodextrin aggregation number, and modified values of the micelle core volume and scattering length density of the micelle core and solvent due to presence of cyclodextrins. The coefficient of correlation $\mathrm{R}^{2}$ was higher than 0.993 for fittings at all the concentrations, except for the case of the $2 \mathrm{mM} \mathrm{AOT}$ data (below CMC, and thus high noise/signal ratio), further demonstrating the quality of the fits reported here. 


\section{Results and discussion}

\section{Sodium bis(2-ethylhexyl) sulfosuccinate micelles in $\mathrm{D}_{2} \mathrm{O}$}

The CMC of AOT in water has been obtained using various techniques, ${ }^{1,14-15,43}$ however, only one publication has reported detailed AOT micelle structure and inter-micelle interactions. ${ }^{2}$ SANS data at the AOT concentrations utilized here have not been previously reported. Hence, we first consider AOT aqueous solutions at different surfactant concentrations to assess changes in the micelle structure and interactions. The results in the absence of cyclodextrins will serve as a reference in order to quantify changes caused by the addition of cyclodextrins.

SANS profiles for AOT micelles at different concentrations in $\mathrm{D}_{2} \mathrm{O}$ are shown in Figure 3. The overall scattering intensity increases with a rise in the surfactant concentration, and a peak in the intensity profile emerges due to higher electrostatic repulsions between the micelles which are expressed in the structure factor $S(q)$. Fits to the experimental SANS data are shown as solid lines in Figure 3 (a) and (b). Table 2 lists some of the important parameters describing micelle structure and interactions extracted by fits to the SANS data.

With an increase in the AOT concentration in water, the micelles grow in size. The surfactant aggregation number increases by $127 \%$ upon $\sim 20 \mathrm{mM}$ increase in AOT concentration. The growth of the micelles is also evident by $\sim 31 \%$ increase in the equivalent micelle diameter $\sigma$ and by $\sim 66 \%$ increase in the overall surface area of a micelle (Table 2 ). On the other hand, the surface area per surfactant head group of a micelle decreases with increase in AOT concentration which indicates denser surfactant packing in a micelle at higher surfactant concentrations. Similar decreasing trends of specific surface area of the micelles (S/V) also support denser packing of the micelles at higher surfactant concentrations. The micelle shape also changes from spherical (axis ratio $\sim 1$ ) to more elongated oblate (axis ratio $>1$ ) as shown in Table 2.

The surfactant aggregation number is plotted in Figure 4 versus the square root of the mole fraction of the surfactant participating in micelles. The aggregation numbers increase linearly with the square root of the surfactant mole fraction, following closely the Ladder model for 
micellization. ${ }^{44}$ Similar dependence of the aggregation number on the surfactant concentration has been reported for AOT, sodium alkyl sulfate, and sodium dodecyl-ortho-xylenesulfonate surfactants. ${ }^{2,45-46}$ The aggregation number $(\sim 15)$ that we obtained at the CMC is in good agreement with the value reported previously. ${ }^{2}$ The aggregation number for AOT micelles in aqueous solutions at $22.5 \mathrm{mM}$ AOT also agrees well with the reported value of $31 .^{2}$ Similarly, the increase of $\sim 6 \%$ in the aggregation number and $\sim 0.59 \%$ in the fractional charge per $1 \mathrm{mM}$ AOT added agree with the reported values of $\sim 4 \%$ and $\sim 0.57 \%$, respectively. ${ }^{2}$

The electrostatic interactions between micelles also increase with an increase of the surfactant concentration in solution, as indicated by higher values of coupling constants and surface potential of the micelles (Table 2). Shorter Debye screening length along with higher repulsions allow micelles to arrange themselves closer to each other in a more ordered lattice-like structure as reflected in periodic oscillations observed in the structure factor (Figure 5). As shown in Figure 5, the structure factor increases from its compressibility limit $S(0)$ while the form factor decreases with an increase in the wavevector $q$ which is typically manifested by the appearance of interaction (correlation) peak in the overall intensity profile. Further, the observed decrease in $S(0)$ value with increase in surfactant concentration indicates lower compressibility of the medium because of increased repulsions between the micelles.

It is evident from the above analysis that, upon increase in AOT concentration in water, the AOT micelles grow in overall size, change their shape from spherical to more elongated oblates, interact more and arrange themselves in smaller inter-micelle distances.

\section{Dioctyl sulfosuccinate micelles in $\mathrm{D}_{2} \mathrm{O}$ in the presence of cyclodextrins}

Having established the AOT micelle structure and inter-micelle interactions in $\mathrm{D}_{2} \mathrm{O}$ in the absence of cyclodextrins, we now address the effect of cyclodextrin addition. In what follows, we first discuss the effects of $\alpha$-cyclodextrin on the AOT micelle structure and interactions. We then study the effects of hydroxypropyl $\beta$-cyclodextrin and compare them to the corresponding effects observed for $\alpha$-CD-AOT system. To the best of our knowledge, SANS studies on AOT in the presence of cyclodextrins have not been previously reported in the literature. 


\section{Effect of a-cyclodextrin on AOT micelle structure and inter-micelle interactions}

SANS data for aqueous AOT solutions at different $\alpha-C D$ concentrations are shown in Figure 6 (a) and (b). At low CD concentrations, a peak is evident in the SANS data due to micelle-micelle repulsive interactions. From the location of the peak maximum $q_{\max }$ the inter-micelle distance can be estimated using $d=2 \pi / q_{\max }$. The peak maximum does not shift much at different $\alpha$-CD concentrations, indicating almost no effect on inter-micelle distances $(d)$ and thereby micelle number densities $\left(N_{P}\right)$ upon $\alpha-\mathrm{CD}$ addition at a fixed AOT concentration. However, the peak intensity decreases strongly with $\alpha-C D$ addition, which indicates weaker electrostatic repulsion between the micelles and lower micelle aggregation numbers. The broadening of the peak also points towards weaker electrostatic repulsions upon cyclodextrin addition. Above $24 \mathrm{mM} \alpha-\mathrm{CD}$ ( $C_{A O T} / C_{\alpha-C D}>2$ ), the scattering profile does not change significantly which indicates the presence of smaller surfactant-cyclodextrin complexes formed upon micelle dissolution. Note that the scattering in this case is still higher than that from $2 \mathrm{mM}$ AOT aqueous solution in the absence of CD.

The effects of $\alpha-C D$ addition on micelle dimensions and inter-micelle interactions were quantified by fitting the SANS data with the form and structure factors described previously. Table 3 summarizes the important parameters related to structure and composition of the AOT micelles in the presence of cyclodextrins obtained from the fittings. Increasing the $\alpha-C D$ concentration from 0 to $40 \mathrm{mM}$ in $12 \mathrm{mM}$ AOT solutions leads to a decrease of the micelle aggregation number by $\sim 65 \%$, eventually leading to the destruction of these micelles. Correspondingly, $\mathrm{CD}$ addition causes a reduction in the overall size of micelles as evident by decreasing values of the effective micelle diameter $\sigma$ and shell thickness $\delta$. Further, the micelle shape changes from an elongated oblate to a sphere upon addition of $\alpha-\mathrm{CD}$ up to $40 \mathrm{mM}$. The average surface area per head group of a micelle rises by $\sim 48 \%$ from its initial value of $\sim 189 \AA^{2}$ with increase in $\alpha-C D$ concentration up to $40 \mathrm{mM}$. Changes in surface area per surfactant head group in a micelle clearly suggest less dense packing at higher $\alpha-C D$ concentrations. 
Together with the changes in the micelle size and shape, the micelle composition also changes. A fraction of cyclodextrins localize in the micelle core upon $\mathrm{CD}$ addition to the surfactant solution. The number of $\mathrm{CD}$ molecules per micelle $(k)$ increases up to about $4 \mathrm{CD}$ molecules per micelle at $12 \mathrm{mM} \mathrm{CD}$, but then decreases to $\sim 1$ at $40 \mathrm{mM} \mathrm{CD}$ (Table 3). The ratio of surfactant to cyclodextrin in the micelles decreases to $\sim 4$ at $12 \mathrm{mM} \alpha-\mathrm{CD}$ with $C_{A O T} / C_{\alpha-C D}=1$, and again increases to $\sim 8$ at $40 \mathrm{mM} \alpha$-CD with $C_{A O T} / C_{\alpha-C D}=0.3$ when $\alpha$-CD is present in excess after forming 1:1 complexes with all the surfactant. Hence, partitioning of CDs between the micelle and solution phases depends on the relative concentration of surfactant and cyclodextrin.

The volume fraction of the micelles decreases continuously with an increase in the $\alpha$-CD concentration as shown in Table 3. Using the volume fraction of the micelles and the total surfactant concentration, one can calculate the amount of surfactant molecules not participating in micelles and, thereby, can obtain the critical micelle concentration of the system. Specifically, the effective $C M C_{S A N S}$ for a given system can be calculated given the total surfactant concentration $C_{A O T}$, micelle volume fraction $\phi$, volume of a micelle $V_{T O T}$ and surfactant aggregation number $\eta$ using the relation $C M C_{S A N S}=C_{A O T}-1 \times 10^{30} \eta \phi /\left(N_{A V} V_{T O T}\right) . N_{A V}$ is Avogadro's number. $C M C_{S A N S}$ values (Table 3) increase drastically by $\sim 220 \%$ by increase in $\alpha$ $\mathrm{CD}$ concentration to $40 \mathrm{mM}$, which clearly indicates the enhancement in the solubility of AOT in $\mathrm{D}_{2} \mathrm{O}$ when $\alpha-\mathrm{CD}$ is present.

The presence of $\alpha-\mathrm{CD}$ also reduces the charge on a micelle and thereby affects the electrostatic interactions between the micelles as reflected by the observed reduction in the coupling constant $\Gamma_{k}$ (Table 3). The electrostatic surface potential decreases by $\sim 44 \%$ from a value of $67.6 \mathrm{mV}$ when $\alpha-C D$ increased to $40 \mathrm{mM}$. On the other hand, the Debye length of the solution increases upon cyclodextrin addition (as shown in Table 3). Because of the decrease in electrostatic interactions, micelles can arrange themselves more randomly, losing their lattice-like arrangement, upon cyclodextrin addition as evident from the disappearance of the peaks and oscillations observed in inter-micelle structure factors at high $\alpha$-CD concentrations (Figure 7). However, the inter-micelle distance remains almost constant irrespective of $\alpha$-CD concentration in the solution as seen by the lack of change in the location of the peak maximum in SANS data 
(Figure 6). Although the total charge on a micelle $Z$ also decreases by $\sim 64 \%$, the value of the degree of ionization $\alpha$ of the surfactants present in a micelle remains almost constant. Since the fractional charge on a micelle remains unchanged upon $\alpha-C D$ addition (Table 3 ), the observed reduction in the micelle charge upon $\mathrm{CD}$ addition can be explained by the decrease in number of surfactant headgroups rather than a decrease in counter-ion dissociation.

In summary, addition of $\alpha-C D$ to aqueous solutions of AOT delays the onset of micellization, affects micelle shape and composition, and reduces inter-micelle interactions. The data extracted from SANS fits indicate that the micelle structure and interactions are almost the same for aqueous solutions of (i) $6 \mathrm{mM}$ AOT in the absence of CD and (ii) $12 \mathrm{mM}$ AOT in the presence of $6 \mathrm{mM} \alpha-\mathrm{CD}$, suggesting 1:1 complexation between $\alpha-\mathrm{CD}$ and AOT. It is interesting to note that the CMC increases from $2.7 \mathrm{mM}$ to $5 \mathrm{mM}$ upon $\mathrm{CD}$ addition, which indicates the solubilization of $5 \mathrm{mM}$ AOT in the solution in the form of complexes, while the rest of the complexes $(1 \mathrm{mM})$ partition in the micelles and affect the micelle size and micelle number density. At low CD concentrations, a small fraction of $\alpha$-CD-AOT complexes may localize in the micelle core, however, at high concentrations CD cause the disassembly of micelles. Addition of $\alpha-C D$ up to the stoichiometric proportion with respect to the total surfactant present in the solution leads to an increase in the number of CD per surfactant molecules present in an AOT micelle up to a value of 0.25 , which is similar to what has been observed for the single-tail anionic surfactant $\mathrm{SDS}$ in a study recently reported from our group (increase up to $\sim 0.27 \mathrm{CD} / \mathrm{SDS}$ at $25 \mathrm{mM}$ SDS). ${ }^{32}$ However, at concentration ratios $\mathrm{C}_{\mathrm{CD}} / \mathrm{C}_{\text {Surfactant }}>1$, the number of $\mathrm{CD}$ molecules localized per surfactant molecule in AOT micelles decreases from 0.25 to 0.12 , whereas, in the case of SDS micelles, this number continues to increase and reaches a value of 0.4.

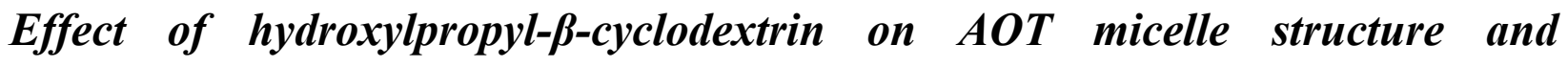 interactions}

Figure 8 shows SANS profiles from $12 \mathrm{mM}$ AOT solutions in $\mathrm{D}_{2} \mathrm{O}$ as affected by the presence of HP- $\beta$-CD. Similar to the case of $\alpha-C D$, a peak is evident in the scattering profiles, reflecting electrostatic interactions, and the intensity of the peak decreases with an increasing HP- $\beta-C D$ concentration. However, the location of the peak maximum shifts towards higher q values with HP- $\beta$-CD addition, instead of no shift observed upon $\alpha-C D$ addition. In particular, addition of up 
to $20 \mathrm{mM}$ HP- $\beta$-CD shifts the peak maxima from $\mathrm{q}=0.051 \AA^{-1}$ to $\mathrm{q}=0.056 \AA^{-1}$. This shift indicates lower inter-micelle distances, and points towards higher number densities of AOT micelles upon addition of HP- $\beta-C D$, similar to the case of SDS and HP- $\beta-C D$ aq. solutions ${ }^{32}$.

Important parameters describing micelle structure and interactions obtained from the fits to SANS data are shown in Table 4. Addition of HP- $\beta-C D$ reduces the aggregation number of AOT micelles by $\sim 3.5 \%(\sim 1.56)$ per $\mathrm{mM}$ of HP- $\beta$-CD added, almost double compared to $\sim 1.6 \%$ $(\sim 0.72)$ per $\mathrm{mM}$ of $\alpha$-CD addition as shown in Figure 9 (a). The reduction in surfactant aggregation numbers causes a decrease in the micelle size (by $28 \%$ upon $20 \mathrm{mM} \mathrm{HP-} \beta-\mathrm{CD}$ ) and changes the micelle shape from oblate to spheres as indicated by the axis ratios. The switch in micelle shape from elongated oblate to sphere occurs at $\sim 12 \mathrm{mM} \mathrm{HP}-\beta-\mathrm{CD}$, half of that $(\sim 24$ $\mathrm{mM}$ ) needed for the case of $\alpha$-CD. Compared to an increase of $\sim 1.2 \%$ per $\mathrm{mM}$ of $\alpha$-CD added, the surface area per headgroup in an AOT micelle increases more prominently $(\sim 3.2 \%$ per $\mathrm{mM}$ of HP- $\beta-C D$ added) showing the higher potency of HP- $\beta-C D$ in affecting the AOT micelle structure compared to $\alpha-\mathrm{CD}$. Smaller values of surfactant aggregation numbers in the presence of HP- $\beta$-CD compared to $\alpha-C D$ can be explained by a corresponding higher solubility of the surfactants in the presence of HP- $\beta-\mathrm{CD}$ compared to $\alpha-\mathrm{CD}$, attributed to the larger cavity size of HP- $\beta-C D$ which can possibly encapsulate both hydrophobic tails of the double-tail AOT, compared to the $\alpha-\mathrm{CD}$ cavity which can capture one of the AOT tails. For example, the CMC value of AOT increases by $\sim 9 \%$ per $\mathrm{mM}$ of HP- $\beta$-CD added (Table 4 ) compared to $\sim 6 \%$ found per $\mathrm{mM}$ of $\alpha$-CD added (Table 3 ) indicating higher solubility of AOT molecules in solution.

The partitioning of HP- $\beta$-CD between AOT micelles and solution is similar to that of $\alpha-\mathrm{CD}$. As seen for the case of $\alpha-\mathrm{CD}$, the aggregation number of cyclodextrins $(k)$ increases at $\mathrm{C}_{\mathrm{CD}}<\mathrm{CMC}$ but it then decreases at high relative $\mathrm{CD}$ concentration (Table 4). The ratio of surfactant to cyclodextrin in micelles is $\sim 2$ at $9 \mathrm{mM} \mathrm{HP}-\beta-\mathrm{CD}$ with $C_{A O T} / C_{H P-\beta-C D}=1.3$, and $\sim 3.3$ at $20 \mathrm{mM}$ HP- $\beta$-CD with $C_{A O T} / C_{H P-\beta-C D}=0.6$. It is evident that the maximum extent of localization of cyclodextrins relative to surfactant molecules in an AOT micelle occurs at higher total concentration ratios of surfactant to cyclodextrin for HP- $\beta-\mathrm{CD}$ compared to $\alpha-\mathrm{CD}$, which may be attributed to a less preferred localization of the bulkier HP- $\beta$-CD molecules in the micelle 
interior compared to $\alpha-\mathrm{CD}$ molecules. A similar trend of increase in the ratio of cyclodextrin to surfactant molecules present in a micelle has been observed for SDS micelles in the presence of HP- $\beta$-CD at lower total concentration $C D /$ surfactant ratio, however, instead of a decrease in the ratio that is observed for $\mathrm{AOT}$ micelles at higher total $\mathrm{CD}$ /surfactant ratio, for SDS micelles the $\mathrm{CD} / \mathrm{SDS}$ in the micelles continues to increase. ${ }^{32}$ To the best of our knowledge, CD-AOT binding ratios in the presence of micelles and the variation in them at different $\mathrm{CD} / \mathrm{AOT}$ concentrations have not been reported previously.

Unlike the constant values of the degree of dissociation of micelle $\alpha$ observed in the case of $\alpha$ $\mathrm{CD}$, addition of HP- $\beta$-CD leads to somewhat higher values of $\alpha$ as shown in Figure 9 (b) and Table 4. The higher degree of dissociation of micelle can be attributed to a lower slope of decrease for charge on a micelle compared to the slope of decrease for surfactant aggregation number as the HP- $\beta-C D$ concentration increases. Higher values of $\alpha$ in case of HP- $\beta-C D$ indicate higher amounts of counterions in the solution which may stabilize the rate of increase in the Debye length to $\sim 1.3 \%$ per $\mathrm{mM}$ of HP- $\beta$-CD added as compared to the $\sim 1.6 \%$ increase per $\mathrm{mM}$ of $\alpha$-CD added.

Similar to $\alpha-C D$, addition of HP- $\beta-C D$ drastically weakens the inter-micelle interactions. As shown in Table 4 , the electrostatic coupling constant $\Gamma_{k}$ decreases by $\sim 56 \%$ upon increasing HP$\beta$-CD concentration to $20 \mathrm{mM}$, which is about double the $\sim 30 \%$ decrease observed when the $\alpha$ $\mathrm{CD}$ concentration increased to $20 \mathrm{mM}$. Figure 9 (c) clearly shows a higher slope of decrease in the coupling constant upon HP- $\beta$-CD addition compared to the slope observed for the $\alpha$-CD case. Accordingly, the surface potential on a micelle also shows a decrease of $\sim 37 \%$ upon HP- $\beta$-CD addition from its value of $67.6 \mathrm{mV}$ found in the absence of $\mathrm{CD}$. The reduction in the charge on a micelle upon HP- $\beta-C D$ addition is mainly because of the decrease in the number of headgroups despite an increase in counter-ion dissociation.

From the above analysis, it is evident that the addition of HP- $\beta-C D$ can affect AOT micelles to a greater extent compared to $\alpha-C D$. An investigation of SDS micelles by our group has also concluded on the higher capacity of HP- $\beta-C D$ in influencing SDS micelle properties compared to $\alpha-C D .{ }^{32}$ In the case of SDS micelles, we have attributed the higher effectiveness of HP- $\beta-C D$ 
compared to $\alpha-\mathrm{CD}$ to the difference in the preferred complexation ratio. HP- $\beta$-CD forms mainly 1:1 complexes with SDS while $\alpha-C D$ forms both 1:1 and 2:1 complexes with SDS, thus HP- $\beta$ $\mathrm{CD}$ can bind with a higher amount of SDS compared to $\alpha-C D$. Since $\alpha-C D$ can apparently form only 1:1 complexes ${ }^{34}$ with AOT, while it can form both 1:1 and 2:1 complexes with SDS, the effectiveness of $\alpha-C D$ in modulating AOT micelles is higher compared to the SDS micelles. For example, at $\mathrm{C}_{\alpha-\mathrm{CD}}=\mathrm{C}_{\text {Surfactant, }}$, the slope of decrease in the aggregation number per unit increase in the $\alpha$-CD concentration is $\sim 3.5 \%$ for AOT micelles, higher (double) than that observed for SDS micelles, $\sim 1.8 \%$. Moreover, in the case of AOT, both $\alpha-C D$ and HP- $\beta$-CD form 1:1 complexes $^{34}$ and hence the higher effectiveness of HP- $\beta$-CD can be ascribed to the difference in encapsulation capabilities between the two types of CDs. $\alpha$-CD can tightly encapsulate one of the hydrophobic tails of AOT, while the other tail may not fit well in the cavity and continue to exhibit some hydrophobicity. HP- $\beta-C D$, on the other hand, may encapsulate both tails of an AOT molecule, resulting in a higher reduction in hydrophobicity of the surfactant in the micelle, and hence affecting the micelle structure to a larger extent. 


\section{Conclusions}

Small-angle neutron scattering has been utilized here to characterize micelles of the anionic double-tail surfactant sodium bis(2-ethylhexyl) sulfosuccinate, commonly known as DOSS or AOT. Micelles formed by AOT in water can be represented as core-shell oblate ellipsoids that interact electrostatically. ${ }^{2}$ Micelles grow in overall size, change their shape from spherical to more elongated oblates, and interact stronger upon increasing the AOT concentration in the aqueous solutions.

Addition of cyclodextrins to the AOT solution leads to the formation of 1:1 CD:AOT inclusion complexes. ${ }^{34}$ Since complexation with CDs reduces the hydrophobicity of the surfactant molecules, the majority of the inclusion complexes formed will not participate in micelles, but will rather remain in solution and elevate the effective critical micelle concentration of the system. However, a small fraction of these inclusion complexes may form mixed micelles with uncomplexed surfactant. As the equilibrium of the surfactant between the micelle and solution changes upon $\mathrm{CD}$ addition, the amount of surfactant molecules in each of the micelles decreases. The surfactant aggregation number decreases at a slope of about 1.56 per $1 \mathrm{mM}$ of HP- $\beta-\mathrm{CD}$, approximately double compared to 0.72 per $1 \mathrm{mM} \alpha-\mathrm{CD}$ addition. The micelles become smaller and change their shape from ellipsoidal towards spherical. Further, the inter-micelle electrostatic interactions weaken owing to a decrease in the surface potential or, equivalently, charge on them. Compared to $\alpha-\mathrm{CD}, \mathrm{HP}-\beta-\mathrm{CD}$ addition causes a higher slope of decrease in the electrostatic coupling. Further increase in the $\mathrm{CD}$ concentration will eventually cause all the AOT micelles to disassociate. Since both HP- $\beta-C D$ and $\alpha-C D$ form primarily 1:1 CD:AOT complexes, the higher effectiveness of HP- $\beta-C D$ in influencing AOT micelles may be attributed to the larger size of HP- $\beta-C D$ compared to $\alpha-C D$, and to the lower hydrophobicity of the HP- $\beta-C D-A O T$ complexes, where both hydrophobic tails of AOT can be accommodated in a single CD cavity, whereas, in complexes formed between AOT and $\alpha-\mathrm{CD}$, one tail can be in the cavity and the other outside.

Similar effects of cyclodextrins on the properties of sodium dodecyl sulfate (SDS) micelles have been reported recently. ${ }^{32-33}$ HP- $\beta-C D$ has higher capacity in influencing SDS micelles and inter- 
micelle interactions compared to $\alpha-\mathrm{CD} \cdot{ }^{32-33}$ Compared to the SDS micelles, the effectiveness of $\alpha-\mathrm{CD}$ in modulating AOT micelles is higher because $\alpha-\mathrm{CD}$ can form only 1:1 complexes with AOT while it can form both 1:1 and 2:1 complexes with SDS which allows $\alpha$-CDs to bind with higher number of AOT molecules compared to SDS molecules at the same concentration.

To the best of our knowledge, SANS studies on AOT-CD-water systems have not been reported in the literature yet. Our study presents the effect of cyclodextrin addition on structure and intermicelle interactions of AOT micelles in $\mathrm{D}_{2} \mathrm{O}$ and investigates how the cyclodextrin type affects the modulation of these micelles. Further, analysis of the SANS data using different scenarios supports the partitioning of a fraction of cyclodextrins in the micelles. The study elucidates the micelle disassociation mechanism upon cyclodextrin addition.

An improved understanding on the modulating mechanism of CDs on micelle structure enables better design of emulsifiers or dispersants in formulations for controlled release of actives. For example, in microemulsion concentrates (MEC) containing hydrophobic actives along with AOT emulsifier, the loss of actives by AOT micelles in the aqueous media upon dilution can be reduced by the addition of appropriate amount of cyclodextrin in the aqueous phase to prevent the micelle formation. The current study provides a guide to the $\mathrm{CD} /$ surfactant ratio to modulate micelle formation and helps in the selection of more effective $\mathrm{CD}$ types for a given application.

\section{Acknowledgements}

We acknowledge the support of the National Institute of Standards and Technology (NIST), Gaithersburg, MD, in providing the neutron research facilities used in this work. We also acknowledge funding from the Gulf of Mexico Research Initiative (GoMRI). 


\section{References}

1. Mukherjee, K.; Moulik, S. P.; Mukherjee, D. C., Thermodynamics of Micellization of Aerosol OT in Polar and Nonpolar Solvents. A Calorimetric Study. Langmuir 1993, 9 (7), 1727-1730.

2. Sheu, E. Y.; Chen, S. H.; Huang, J. S., Structure and Growth of Bis( 2-Ethylhexyl) Sulfosuccinate Micelles in Aqueous Solutions. Journal of Physical Chemistry 1987, 91 (12), 3306-3310.

3. Kaler, E. W.; Billman, J. F.; Fulton, J. L.; Smith, R. D., A Small-Angle Neutron-Scattering Study of Intermicellar Interactions in Microemulsions of AOT, Water, and near-Critical Propane. Journal of Physical Chemistry 1991, 95 (1), 458-462.

4. $\quad$ Nave, S.; Eastoe, J.; Heenan, R. K.; Steytler, D.; Grillo, I., What Is So Special About AerosolOT? 2. Microemulsion Systems. Langmuir 2000, 16 (23), 8741-8748.

5. $\quad$ Chen, S. H.; Chang, S. L.; Strey, R., Structural Evolution within the One-Phase Region of a 3Component Microemulsion System - Water-N-Decane-Sodium-Bis-Ethylhexylsulfosuccinate (AOT). Journal of Chemical Physics 1990, 93 (3), 1907-1918.

6. Alexandridis, P.; Holzwarth, J. F.; Hatton, T. A., Thermodynamics of Droplet Clustering in Percolating AOT Water-in-Oil Microemulsions. Journal of Physical Chemistry 1995, 99 (20), 8222-8232.

7. Kotlarchyk, M.; Huang, J. S.; Chen, S. H., Structure of AOT-Reversed Micelles Determined by Small-Angle Neutron-Scattering. Journal of Physical Chemistry 1985, 89 (20), 4382-4386.

8. Mackay, D.; Hossain, K., Interfacial Tensions of Oil, Water, Chemical Dispersant Systems. Canadian Journal of Chemical Engineering 1982, 60 (4), 546-550.

9. Tansel, B.; Lee, M.; Berbakov, J.; Tansel, D. Z.; Koklonis, U., Dispersion of Louisiana Crude Oil in Salt Water Environment by Corexit 9500a in the Presence of Natural Coastal Materials. Estuarine, Coastal and Shelf Science 2014, 143 (20), 58-64.

10. Riehm, D. A.; McCormick, A. V., The Role of Dispersants' Dynamic Interfacial Tension in Effective Crude Oil Spill Dispersion. Marine Pollution Bulletin 2014, 84 (1-2), 155-163.

11. Rodham, D. K., Colloid and Interface Science in Formulation Research for Crop Protection Products. Current Opinion in Colloid \& Interface Science 2000, 5 (5-6), 280-287.

12. Changez, M.; Varshney, M., Aerosol-OT Microemulsions as Transdermal Carriers of Tetracaine Hydrochloride. Drug Development and Industrial Pharmacy 2000, 26 (5), 507-512.

13. Dey, J.; Bhattacharjee, J.; Hassan, P. A.; Aswal, V. K.; Das, S.; Ismail, K., Micellar Shape Driven Counterion Binding. Small-Angle Neutron Scattering Study of AOT Micelle. Langmuir 2010, 26 (20), 15802-15806.

14. Nave, S.; Eastoe, J.; Penfold, J., What Is So Special About Aerosol-OT? 1. Aqueous Systems. Langmuir 2000, 16 (23), 8733-8740.

15. Umlong, I. M.; Ismail, K., Micellization of AOT in Aqueous Sodium Chloride, Sodium Acetate, Sodium Propionate, and Sodium Butyrate Media: A Case of Two Different Concentration Regions of Counterion Binding. Journal of Colloid and Interface Science 2005, 291, 529-536.

16. Sarkar, B.; Lam, S.; Alexandridis, P., Micellization of Alkyl-Propoxy-Ethoxylate Surfactants in Water-Polar Organic Solvent Mixtures. Langmuir 2010, 26 (13), 10532-10540.

17. Lin, Y. N.; Alexandridis, P., Cosolvent Effects on the Micellization of an Amphiphilic Siloxane Graft Copolymer in Aqueous Solutions. Langmuir 2002, 18 (11), 4220-4231.

18. Sarkar, B.; Ravi, V.; Alexandridis, P., Micellization of Amphiphilic Block Copolymers in Binary and Ternary Solvent Mixtures. Journal of Colloid and Interface Science 2013, 390 (1), 137-146.

19. Valente, A. J. M.; Söderman, O., The Formation of Host-Guest Complexes between Surfactants and Cyclodextrins. Advances in Colloid and Interface Science 2014, 205 (0), 156-176.

20. Connors, K. A., The Stability of Cyclodextrin Complexes in Solution. Chemical Reviews 1997, 97 (5), 1325-1357.

21. Tsianou, M.; Alexandridis, P., Control of the Rheological Properties in Solutions of a Polyelectrolyte and an Oppositely Charged Surfactant by the Addition of Cyclodextrins. Langmuir 1999, 15 (23), 8105-8112. 
22. Astray, G.; Gonzalez-Barreiro, C.; Mejuto, J. C.; Rial-Otero, R.; Simal-Gándara, J., A Review on the Use of Cyclodextrins in Foods. Food Hydrocolloids 2009, 23 (7), 1631-1640.

23. Kurkov, S. V.; Loftsson, T., Cyclodextrins. International Journal of Pharmaceutics 2013, 453 (1), 167-180.

24. Mosinger, J.; Toma'nkova', V.; Ne`mcova', I.; Zy'ka, J., Cyclodextrins in Analytical Chemistry. Analytical Letters 2001, 34 (12), 1979-2004.

25. Szente, L.; Szejtli, J., Highly Soluble Cyclodextrin Derivatives: Chemistry, Properties, and Trends in Development. Advanced Drug Delivery Reviews 1999, 36, 17-28.

26. Mellet, C. O.; Fernandez, J. M. G.; Benito, J. M., Cyclodextrin-Based Gene Delivery Systems. Chemical Society Reviews 2011, 40 (3), 1586-1608.

27. Brocos, P.; Banquy, X.; Díaz-Vergara, N.; Pérez-Casas, S.; Piñeiro, Á.; Costas, M., A Critical Approach to the Thermodynamic Characterization of Inclusion Complexes: Multiple-Temperature Isothermal Titration Calorimetric Studies of Native Cyclodextrins with Sodium Dodecyl Sulfate. Journal of Physical Chemistry B 2011, 115 (49), 14381-14396.

28. Funasaki, N.; Ishikawa, S.; Neya, S., $1: 1$ and $1: 2$ Complexes between Long-Chain Surfactant and $\alpha$-Cyclodextrin Studied by NMR. Journal of Physical Chemistry B 2004, 108 (28), 9593-9598.

29. Mwakibete, H.; Cristantino, R.; Bloor, D. M.; Wyn-Jones, E.; Holzwarth, J. F., Reliability of the Experimental Methods to Determine Equilibrium-Constatns for Surfactant Cyclodextrin Inclusion Complexes. Langmuir 1995, 11 (1), 57-60.

30. Yunus, W. M. Z. W.; Taylor, J.; Bloor, D. M.; Hall, D. G.; Wyn-Jones, E., Electrochemical Measurements on the Binding of Sodium Dodecyl-Sulfate and Dodecyltrimethylammonium Bromide with $\alpha$ - and $\beta$-Cyclodextrins. Journal of Physical Chemistry 1992, 96 (22), 8979-8982.

31. Fajalia, A. I.; Tsianou, M., Charging and Uncharging a Neutral Polymer in Solution: A SmallAngle Neutron Scattering Investigation. Journal of Physical Chemistry B 2014, 118 (36), 10725-10739.

32. Tsianou, M.; Fajalia, A. I., Cyclodextrins and Surfactants in Aqueous Solution above the Critical Micelle Concentration: Where Are the Cyclodextrins Located? Langmuir 2014, 30 (46), 13754-13764.

33. Fajalia, A. I.; Tsianou, M., Self-Assembly Control Via Molecular Recognition: Effect of Cyclodextrins on Surfactant Micelle Structure and Interactions Determined by SANS. Colloids and Surfaces A: Physicochemical and Engineering Aspects 2014, DOI: 10.1016/j.colsurfa.2014.12.024.

34. Qiu, X. M.; Sun, D. Z.; Wei, X. L.; Yin, B. L., Thermodynamic Study of the Inclusion Interaction between Gemini Surfactants and Cyclodextrins by Isothermal Titration Microcalorimetry. Journal of Solution Chemistry 2007, 36 (3), 303-312.

35. Palepu, R.; Richardson, J. E.; Reinsborough, V. C., Binding Constants of BetaCyclodextrin/Surfactant Inclusion by Conductivity Measurements. Langmuir 1989, 5 (1), 218-221.

36. Kline, S. R., Reduction and Analysis of SANS and USANS Data Using Igor Pro. Journal of Applied Crystallography 2006, 39, 895-900.

37. Hansen, J. P.; Hayter, J. B., A Rescaled MSA Structure Factor for Dilute Charged Colloidal Dispersions. Molecular Physics 1982, 46 (3), 651-656.

38. Hayter, J. B.; Penfold, J., An Analytic Structure Factor for Macroion Solutions. Molecular Physics 1981, 42 (1), 109-118.

39. Sheu, E. Y.; Chen, S. H., Thermodynamic Analysis of Polydispersity in Ionic Micellar Systems and Its Effect on Small-Angle Neutron Scattering Data Treatment. The Journal of Physical Chemistry 1988, 92 (15), 4466-4474.

40. Bendedouch, D.; Chen, S. H.; Koehler, W. C., Determination of Interparticle Structure Factors in Ionic Micellar Solutions by Small-Angle Neutron-Scattering. Journal of Physical Chemistry 1983, 87 (14), 2621-2628.

41. Bendedouch, D.; Chen, S. H., Effect of an Attractive Potential on the Interparticle Structure of Ionic Micelles at High Salt Concentration. Journal of Physical Chemistry 1984, 88 (4), 648-652.

42. Kotlarchyk, M.; Chen, S.-H., Analysis of Small Angle Neutron Scattering Spectra from Polydisperse Interacting Colloids. Journal of Chemical Physics 1983, 79 (5), 2461-2469. 
43. Pal, M. K.; Pal, P. K., Dichroic Probe of Different Modes of Aggregation of Pinacyanol by Bis(2Ethylhexyl)Sulfosuccinate Below and above Its Critical Micelle Concentration. Journal of Physical Chemistry 1990, 94 (6), 2557-2559.

44. Missel, P. J.; Mazer, N. A.; Benedek, G. B.; Young, C. Y.; Carey, M. C., Thermodynamic Analysis of the Growth of Sodium Dodecyl Sulfate Micelles. Journal of Physical Chemistry 1980, 84 (9), 1044-1057.

45. Sheu, E. Y.; Chen, S. H.; Huang, J. S., Structure, Interaction, and Growth of Sodium DodecylOrtho-Xylenesulfonate Micelles in Aqueous-Solutions. Journal of Physical Chemistry 1987, 91 (6), $1535-1541$.

46. Vass, S., Structure of Sodium Alkyl Sulfate Micelles - Results from Density, Small-Angle Neutron-Scattering, and Positron-Annihilation Measurements. Structural Chemistry 1991, 2 (3-4), 375397.

47. Tanford, C., Micelle Shape and Size. Journal of Physical Chemistry 1972, 76 (21), 3020. 
Table 1. Scattering lengths and parameters used for fitting SANS data of AOT-CD aqueous solutions: $\sum b_{i}$ is the scattering length of species $i$ (sum of the scattering lengths of the atoms present in the group), $v_{i}$ is the molecular volume of species $i$ (molecular volumes of $\mathrm{CH}_{3}$ and $\mathrm{CH}_{2}$ groups are calculated from the Tanford formula ${ }^{47}$ ), $\rho_{i}$ is the scattering length density of species $i$, and $M W_{i}$ is the molecular weight of species $i$.

\begin{tabular}{|lllll|}
\hline Species & $\sum b_{i}(\AA)$ & $v_{i}\left(\AA^{3}\right)$ & $\rho_{i}\left(\AA^{-2}\right)$ & $M W_{i}$ \\
\hline $\mathrm{Na}^{+}$ & $3.63 \mathrm{E}-05$ & 8.99 & $4.04 \mathrm{E}-06$ & 23.0 \\
$\mathrm{OSO}_{2}{ }^{-}$ & $2.02 \mathrm{E}-04$ & 49.80 & $4.06 \mathrm{E}-06$ & 80.1 \\
$\mathrm{D}_{2} \mathrm{O}$ & $1.91 \mathrm{E}-04$ & 30.19 & $6.34 \mathrm{E}-06$ & 20.0 \\
$\mathrm{CH}_{3}$ & $-4.57 \mathrm{E}-05$ & 54.30 & $-8.41 \mathrm{E}-07$ & 15.0 \\
$\mathrm{CH}_{2}$ & $-8.27 \mathrm{E}-06$ & 26.90 & $-3.07 \mathrm{E}-07$ & 14.0 \\
$\alpha-\mathrm{CD}$ & $1.71 \mathrm{E}-03$ & 968.98 & $1.77 \mathrm{E}-06$ & 774.3 \\
$\mathrm{HP}-\beta-\mathrm{CD}$ & $2.45 \mathrm{E}-03$ & 1181.01 & $2.08 \mathrm{E}-06$ & 1381.3 \\
$\mathrm{AOT}$ & $3.80 \mathrm{E}-04$ & 611.00 & $6.23 \mathrm{E}-07$ & 444.6 \\
\hline
\end{tabular}


Table 2. Parameters describing the micelle structure and inter-micelle interactions obtained by fitting SANS data of AOT in $\mathrm{D}_{2} \mathrm{O}$ using the form and structure factors described in the text. $\eta$ is the number of surfactant molecules per micelle, $Z$ the micelle charge, $\alpha$ the fractional charge or charge per surfactant molecule in a micelle, $b$ the minor core radius of a micelle, $\varepsilon$ the ratio of major to minor axis of a micelle, $\delta$ the micelle shell thickness, $\sigma_{0}$ the equivalent spherical diameter of the micelle, $S_{\text {micelle }}$ the surface area of each micelle, $S_{\text {surfactant }}$ the surface area per surfactant molecule in a micelle, $S / V$ the surface area per unit volume of the micelle. $\rho_{C}, \rho_{S h}$ and $\rho_{S}$ are the scattering length densities of the micelle core, micelle shell and solvent, respectively, $\phi$ is the volume fraction of the micelles, $\Gamma_{k}$ the electrostatic coupling constant for the micelles defined as $\Gamma_{k}=4 Z^{2} l_{B} / \sigma_{0}\left(2+\kappa \sigma_{0}\right)^{2}$ where $l_{B}$ is the Bjerrum length, $\kappa^{-1}$ the Debye length of the solvent, $\psi$ the electrostatic surface potential on the micelles, and $d$ the intermicelle distance. Reduced $\chi$ values $^{31}$ were calculated using $\chi=\sqrt{\frac{1}{\left(N-N_{p}+1\right)} \sum_{i=1}^{N}\left[\left(y_{i}-y_{i}^{\mathrm{m}}\right) / \sigma_{i}\right]^{2}}$ where $y_{i}$ represents experimental data and $y_{i}^{m}$ the model prediction, $\sigma_{i}$ is the standard deviation of the measurement, $N$ is the number of data points, and $N_{P}$ the number of fitting parameters. Uncertainties in the values of fitting parameters are shown in parentheses in italics.

\begin{tabular}{|ccccccccccc|}
\hline $\mathrm{C}_{\mathrm{AOT}}(\mathrm{mM})$ & $\eta$ & $\mathrm{Z}$ & $\alpha$ & $a(\AA)$ & $\varepsilon$ & $\delta(\AA)$ & $\sigma_{0}(\AA)$ & $\begin{array}{c}\mathrm{S}_{\text {micelle }} \\
\left(\AA^{-2}\right)\end{array}$ & $\begin{array}{c}\mathrm{S}_{\text {surfactant }} \\
\left(\AA^{-2}\right)\end{array}$ & $\begin{array}{c}S / V \\
\left(\AA^{-1}\right)\end{array}$ \\
\hline 2.0 & $15.0( \pm 1.5)$ & $5.0( \pm 0.0)$ & 0.33 & 12.5 & 1.00 & 4.1 & 33.1 & 3440.2 & 229.3 & 0.18 \\
6.0 & $22.8( \pm 0.1)$ & $7.7( \pm 0.7)$ & 0.34 & 15.4 & 1.22 & 4.6 & 38.0 & 4423.7 & 194.3 & 0.15 \\
12.0 & $27.7( \pm 0.1)$ & $9.4( \pm 0.1)$ & 0.34 & 16.9 & 1.35 & 4.9 & 40.6 & 5227.0 & 188.6 & 0.14 \\
22.5 & $34.0( \pm 0.1)$ & $12.5( \pm 0.1)$ & 0.37 & 18.8 & 1.49 & 5.2 & 43.5 & 5711.4 & 168.0 & 0.13 \\
\hline
\end{tabular}

\begin{tabular}{|ccccccccccc|}
\hline $\mathrm{C}_{\mathrm{AOT}}(\mathrm{mM})$ & $\rho_{\mathrm{C}}\left(\AA^{-2}\right)$ & $\rho_{\mathrm{Sh}}\left(\AA^{-2}\right)$ & $\rho_{\mathrm{S}}\left(\AA^{-2}\right)$ & $\varphi$ & $\kappa^{-1}(\AA)$ & $\Gamma_{\mathrm{k}}$ & $\begin{array}{c}\psi \\
(\mathrm{mV})\end{array}$ & $d(\AA)$ & $\chi$ \\
\hline 2.0 & $3.28 \mathrm{E}-07$ & $6.03 \mathrm{E}-06$ & $6.34 \mathrm{E}-06$ & 0.00031 & 371.7 & 0.19 & 53.2 & 396.3 & 1.28 \\
6.0 & $3.28 \mathrm{E}-07$ & $6.02 \mathrm{E}-06$ & $6.34 \mathrm{E}-06$ & 0.00259 & 126.3 & 0.35 & 65.1 & 223.2 & 1.20 \\
12.0 & $3.28 \mathrm{E}-07$ & $6.03 \mathrm{E}-06$ & $6.34 \mathrm{E}-06$ & 0.00712 & 76.1 & 0.40 & 67.6 & 170.2 & 1.84 \\
22.5 & $3.28 \mathrm{E}-07$ & $6.03 \mathrm{E}-06$ & $6.34 \mathrm{E}-06$ & 0.01517 & 50.3 & 0.45 & 73.6 & 141.5 & 2.87 \\
\hline
\end{tabular}


Table 3. Parameters describing the micelle structure and inter-micelle interactions obtained by fitting SANS data of AOT $\left(12 \mathrm{mM}\right.$ ) in $\mathrm{D}_{2} \mathrm{O}$ with $\alpha-\mathrm{CD}$ at different concentrations (a fraction of $\mathrm{CD}$ may localize in the micelle and the remainder stays in solution) using the form and structure factors described in the text: $k$ is the number of cyclodextrin molecules per micelle, $\Gamma$ the fraction of cyclodextrins localizing in micelles, and $\mathrm{CMC}_{\mathrm{SANS}}$ the apparent critical micelle concentration of AOT. All other parameters are defined in Table 2.

\begin{tabular}{|cccccccccccc|}
\hline $\begin{array}{c}\mathrm{C}_{\alpha-\mathrm{CD}} \\
(\mathrm{mM})\end{array}$ & $\eta$ & $\mathrm{Z}$ & $\alpha$ & $\begin{array}{c}\mathrm{N}_{\mathrm{P}} \\
\left(\AA^{-3}\right)\end{array}$ & $\begin{array}{c}a \\
(\AA)\end{array}$ & $\varepsilon$ & $\begin{array}{c}\delta \\
(\AA)\end{array}$ & $\begin{array}{c}\sigma_{0} \\
(\AA)\end{array}$ & $\begin{array}{c}\mathrm{S}_{\text {surfactant }} \\
\left(\AA^{-2}\right)\end{array}$ & $\begin{array}{c}\mathrm{CMC}_{\text {SANS }} \\
(\mathrm{mM})\end{array}$ & $\chi$ \\
\hline 0 & $27.7( \pm 0.1)$ & $9.4( \pm 0.1)$ & 0.34 & $2.03 \mathrm{E}-07$ & 16.9 & 1.35 & 4.9 & 40.6 & 188.6 & 2.7 & 1.84 \\
6 & $20.7( \pm 0.1)$ & $7.1( \pm 0.1)$ & 0.34 & $2.04 \mathrm{E}-07$ & 16.4 & 1.31 & 4.0 & 38.2 & 223.0 & 5.0 & 1.67 \\
12 & $16.0( \pm 0.1)$ & $5.5( \pm 0.3)$ & 0.34 & $2.04 \mathrm{E}-07$ & 15.0 & 1.20 & 3.6 & 35.5 & 248.4 & 6.6 & 1.01 \\
18 & $13.2( \pm 0.1)$ & $4.5( \pm 0.2)$ & 0.34 & $2.04 \mathrm{E}-07$ & 13.8 & 1.10 & 3.3 & 33.4 & 265.9 & 7.5 & 1.57 \\
24 & $10.4( \pm 0.2)$ & $3.6( \pm 0.6)$ & 0.35 & $2.03 \mathrm{E}-07$ & 12.2 & 1.00 & 3.1 & 30.7 & 284.0 & 8.5 & 2.07 \\
40 & $9.7( \pm 0.1)$ & $3.4( \pm 0.4)$ & 0.35 & $2.11 \mathrm{E}-07$ & 11.4 & 1.00 & 3.2 & 29.4 & 278.6 & 8.6 & 1.19 \\
\hline
\end{tabular}

\begin{tabular}{|ccccccccccc|}
\hline $\begin{array}{c}\mathrm{C}_{\alpha-\mathrm{CD}} \\
(\mathrm{mM})\end{array}$ & $\rho_{\mathrm{C}}\left(\AA^{-2}\right)$ & $\rho_{\mathrm{Sh}}\left(\AA^{-2}\right)$ & $\rho_{\mathrm{S}}\left(\AA^{-2}\right)$ & $\varphi$ & $\Gamma$ & $k$ & $d(\AA)$ & $\begin{array}{c}\kappa^{-1} \\
(\AA)\end{array}$ & $\Gamma_{\mathrm{k}}$ & $\begin{array}{c}\psi \\
(\mathrm{mV})\end{array}$ \\
\hline 0 & $3.28 \mathrm{E}-07$ & $6.03 \mathrm{E}-06$ & $6.34 \mathrm{E}-06$ & 0.00712 & 0.00 & $0.0( \pm 0.0)$ & 170.2 & 76.1 & 0.40 & 67.6 \\
6 & $7.05 \mathrm{E}-07$ & $6.03 \mathrm{E}-06$ & $6.35 \mathrm{E}-06$ & 0.00593 & 0.21 & $3.7( \pm 0.0)$ & 170.0 & 87.7 & 0.32 & 56.1 \\
12 & $8.17 \mathrm{E}-07$ & $6.03 \mathrm{E}-06$ & $6.35 \mathrm{E}-06$ & 0.00478 & 0.11 & $4.0( \pm 0.1)$ & 169.9 & 99.6 & 0.25 & 48.3 \\
18 & $8.43 \mathrm{E}-07$ & $6.03 \mathrm{E}-06$ & $6.36 \mathrm{E}-06$ & 0.00399 & 0.07 & $3.6( \pm 0.1)$ & 169.9 & 109.4 & 0.21 & 43.4 \\
24 & $7.95 \mathrm{E}-07$ & $6.03 \mathrm{E}-06$ & $6.37 \mathrm{E}-06$ & 0.00308 & 0.03 & $2.5( \pm 0.1)$ & 170.1 & 123.1 & 0.16 & 38.4 \\
40 & $6.10 \mathrm{E}-07$ & $6.03 \mathrm{E}-06$ & $6.39 \mathrm{E}-06$ & 0.00281 & 0.01 & $1.2( \pm 0.1)$ & 167.9 & 124.6 & 0.15 & 37.9 \\
\hline
\end{tabular}


Table 4. Parameters describing the micelle structure and inter-micelle interactions obtained by fitting SANS data of AOT $(12 \mathrm{mM})$ in $\mathrm{D}_{2} \mathrm{O}$ with $\mathrm{HP}-\beta-\mathrm{CD}$ at different concentrations (a fraction of $\mathrm{CD}$ may localize in the micelle and the remainder stays in solution) using the form and structure factors described in the text. The various parameters are defined in Table $\mathbf{2}$ and Table $\mathbf{3}$.

\begin{tabular}{|ccccccccccccc}
\hline $\begin{array}{c}\mathrm{C}_{\text {HP- }-\mathrm{CD}} \\
(\mathrm{mM})\end{array}$ & $\eta$ & $\mathrm{Z}$ & $\alpha$ & $\begin{array}{c}\mathrm{N}_{\mathrm{P}} \\
\left(\AA^{-3}\right)\end{array}$ & $\begin{array}{c}a \\
(\AA)\end{array}$ & $\varepsilon$ & $\begin{array}{c}\delta \\
(\AA)\end{array}$ & $\begin{array}{c}\sigma_{0} \\
(\AA)\end{array}$ & $\begin{array}{c}\mathrm{S}_{\text {surfactant }} \\
\left(\AA^{-2}\right)\end{array}$ & $\begin{array}{c}\text { CMC } \\
(\mathrm{mM})\end{array}$ \\
\hline 0 & $27.7( \pm 0.1)$ & $9.4( \pm 0.1)$ & 0.34 & $2.03 \mathrm{E}-07$ & 16.9 & 1.35 & 4.9 & 40.6 & 188.6 & 2.7 & 1.84 \\
3 & $20.8( \pm 0.1)$ & $8.2( \pm 0.1)$ & 0.39 & $2.04 \mathrm{E}-07$ & 17.0 & 1.35 & 3.9 & 38.6 & 228.0 & 5.0 & 2.17 \\
6 & $15.7( \pm 0.1)$ & $6.6( \pm 0.2)$ & 0.42 & $2.11 \mathrm{E}-07$ & 16.3 & 1.30 & 3.2 & 36.4 & 268.5 & 6.5 & 1.28 \\
9 & $11.9( \pm 0.1)$ & $5.2( \pm 0.1)$ & 0.44 & $2.50 \mathrm{E}-07$ & 15.0 & 1.19 & 2.8 & 33.9 & 303.4 & 7.0 & 1.20 \\
12 & $9.0( \pm 0.1)$ & $4.0( \pm 0.2)$ & 0.45 & $3.01 \mathrm{E}-07$ & 12.3 & 1.00 & 2.7 & 30.1 & 316.8 & 7.5 & 1.30 \\
20 & $8.6( \pm 0.1)$ & $3.9( \pm 0.2)$ & 0.45 & $3.12 \mathrm{E}-07$ & 11.7 & 1.00 & 2.8 & 29.1 & 310.2 & 7.6 & 1.65 \\
\hline
\end{tabular}

\begin{tabular}{|ccccccccccc|}
\hline $\begin{array}{c}\mathrm{C}_{\mathrm{HP}-\beta-\mathrm{CD}} \\
(\mathrm{mM})\end{array}$ & $\rho_{\mathrm{C}}\left(\AA^{-2}\right)$ & $\rho_{\mathrm{Sh}}\left(\AA^{-2}\right)$ & $\rho_{\mathrm{S}}\left(\AA^{-2}\right)$ & $\varphi$ & $\Gamma$ & $k$ & $d(\AA)$ & $\begin{array}{c}\kappa^{-1} \\
(\AA)\end{array}$ & $\begin{array}{c}\Gamma_{\mathrm{k}} \\
(\mathrm{mV})\end{array}$ \\
\hline 0 & $3.28 \mathrm{E}-07$ & $6.03 \mathrm{E}-06$ & $6.34 \mathrm{E}-06$ & 0.0071 & 0.00 & $0.0( \pm 0.0)$ & 170.2 & 76.1 & 0.40 & 67.6 \\
3 & $9.26 \mathrm{E}-07$ & $6.03 \mathrm{E}-06$ & $6.35 \mathrm{E}-06$ & 0.0062 & 0.48 & $4.2( \pm 0.1)$ & 170.0 & 81.6 & 0.36 & 63.0 \\
6 & $1.24 \mathrm{E}-06$ & $6.03 \mathrm{E}-06$ & $6.35 \mathrm{E}-06$ & 0.0054 & 0.35 & $6.0( \pm 0.0)$ & 167.9 & 89.5 & 0.30 & 55.0 \\
9 & $1.37 \mathrm{E}-06$ & $6.03 \mathrm{E}-06$ & $6.35 \mathrm{E}-06$ & 0.0051 & 0.26 & $5.7( \pm 0.0)$ & 158.7 & 92.4 & 0.24 & 47.6 \\
12 & $1.20 \mathrm{E}-06$ & $6.03 \mathrm{E}-06$ & $6.36 \mathrm{E}-06$ & 0.0043 & 0.13 & $3.2( \pm 0.1)$ & 149.2 & 95.8 & 0.19 & 42.4 \\
20 & $8.83 \mathrm{E}-07$ & $6.03 \mathrm{E}-06$ & $6.36 \mathrm{E}-06$ & 0.0040 & 0.07 & $2.6( \pm 0.1)$ & 147.4 & 95.9 & 0.18 & 42.6 \\
\hline
\end{tabular}



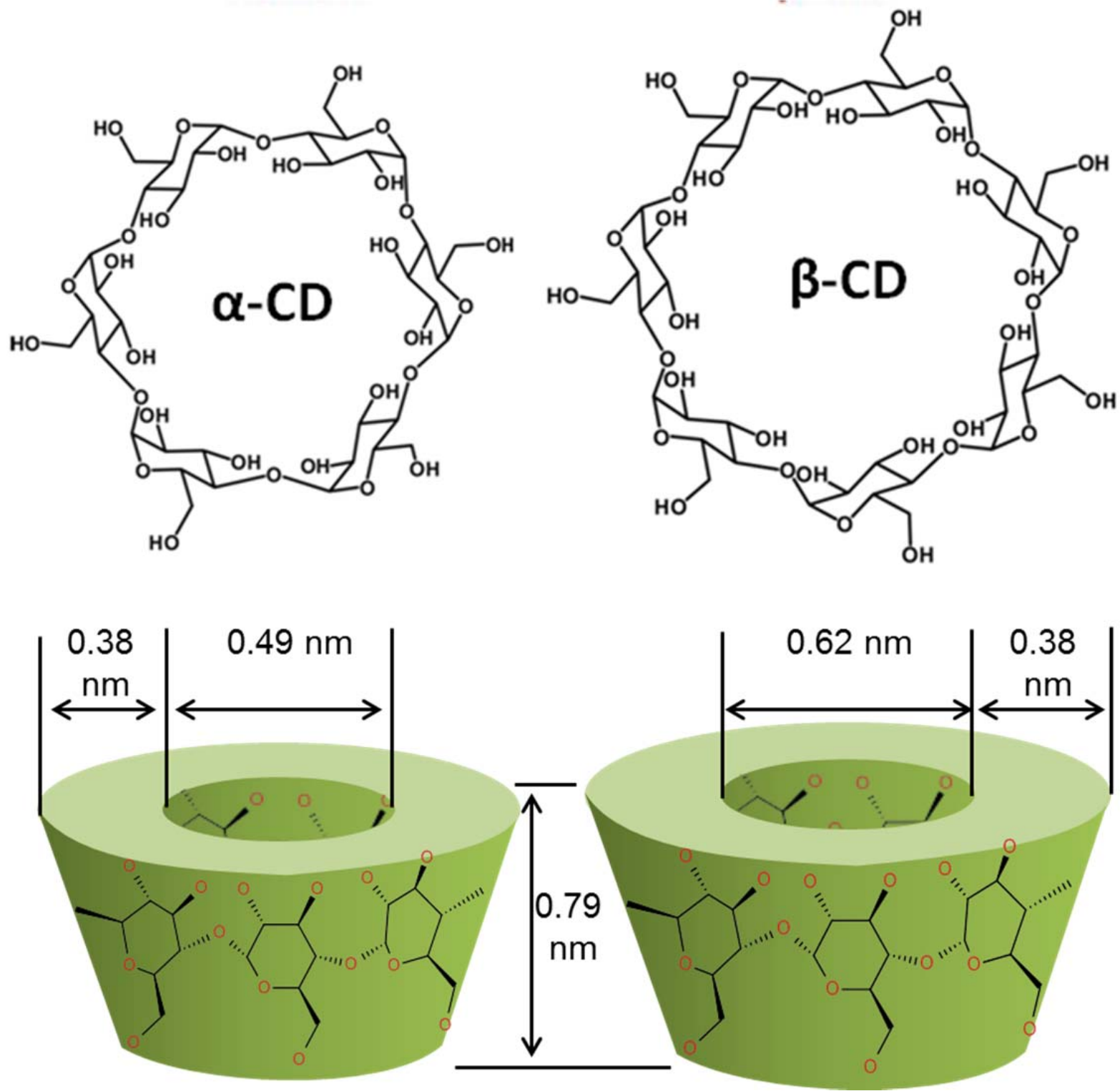

Figure 1. Chemical structure, geometry, and dimensions of $\alpha$ - and $\beta$-cyclodextrin. 


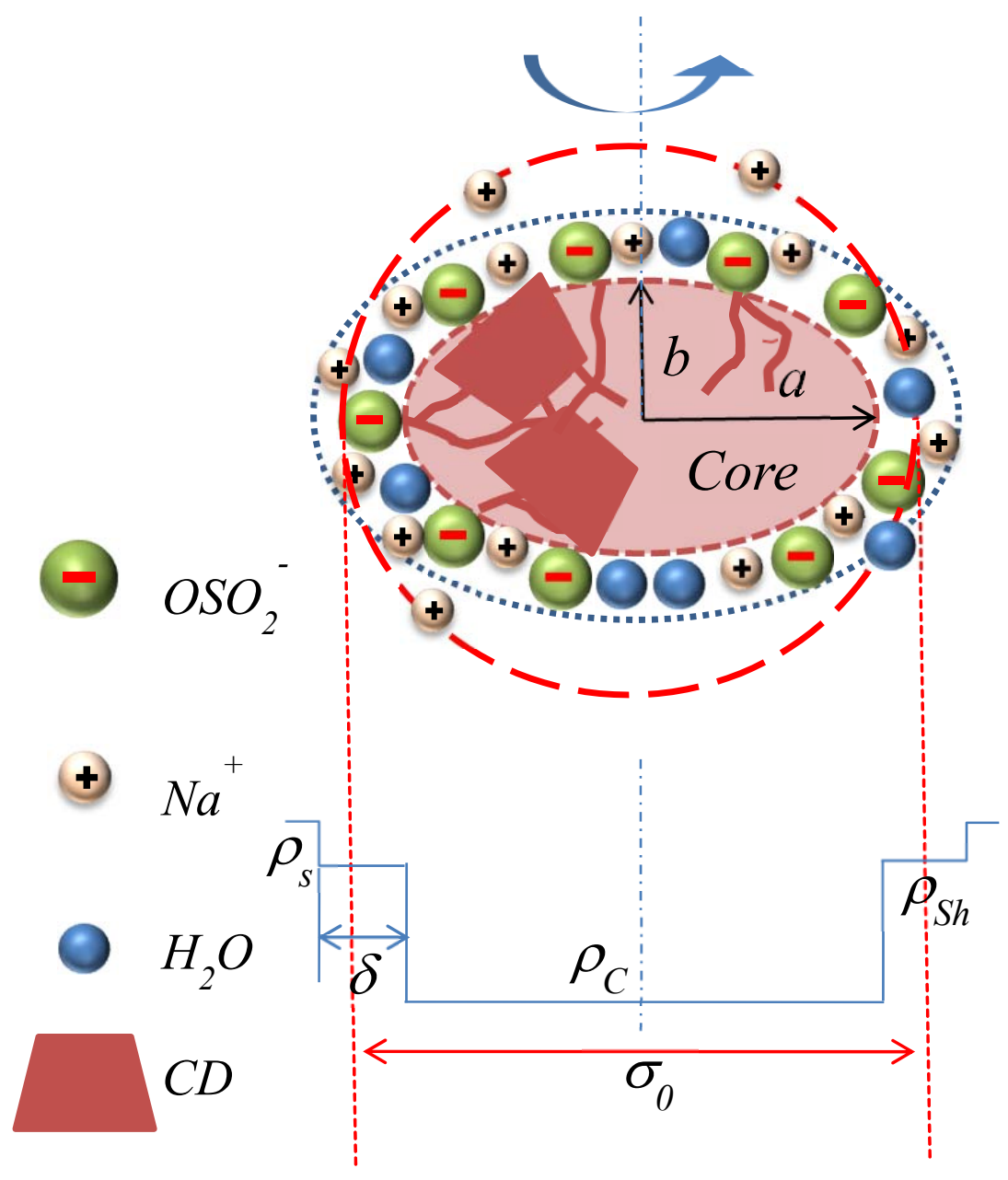

Figure 2. Schematic of a sodium bis(2-ethylhexyl) sulfosuccinate micelle represented as coreshell oblate ellipsoid. The core of the ellipsoid has major axis $a$ and minor axis $b$ and comprises of the hydrophobic alkyl tail of surfactants and some cyclodextrins. The shell has constant thickness of $\delta$ and consists of surfactant head groups, associated water molecules, and counterions (depending on the degree of ionization). $\rho_{C}, \rho_{S h}$ and $\rho_{s}$ are the scattering length density of micelle core, micelle shell and solvent respectively. $\sigma_{0}$ is the equivalent spherical diameter for the ellipsoid. 

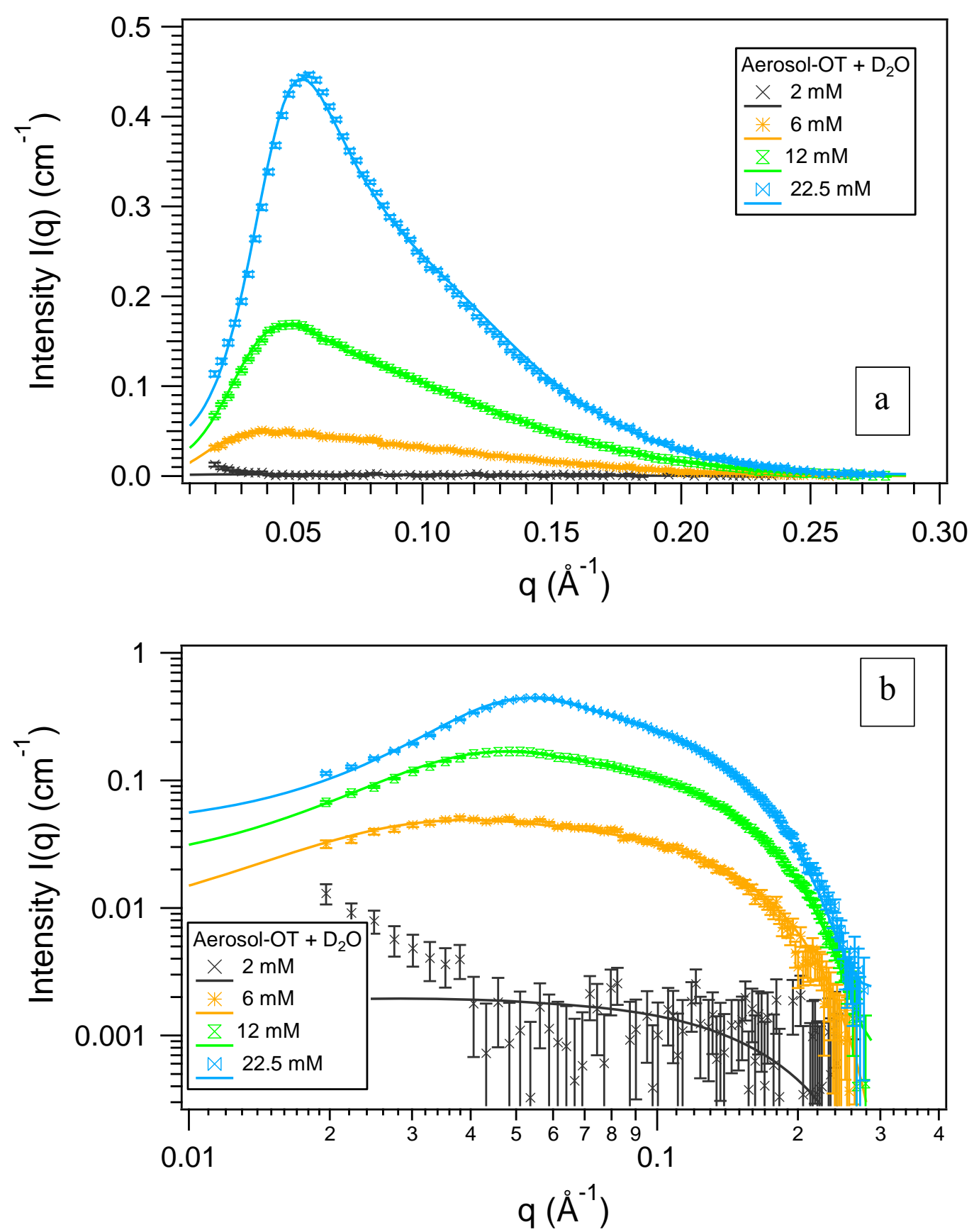

Figure 3. SANS intensity profiles from sodium bis(2-ethylhexyl) sulfosuccinate solutions in $\mathrm{D}_{2} \mathrm{O}$ at different surfactant concentrations: (a) linear plot, (b) log-log plot. Markers represent experimental data and solid lines represent corresponding fits using the core-shell oblate form factor with Hansen-Hayter structure factor. 


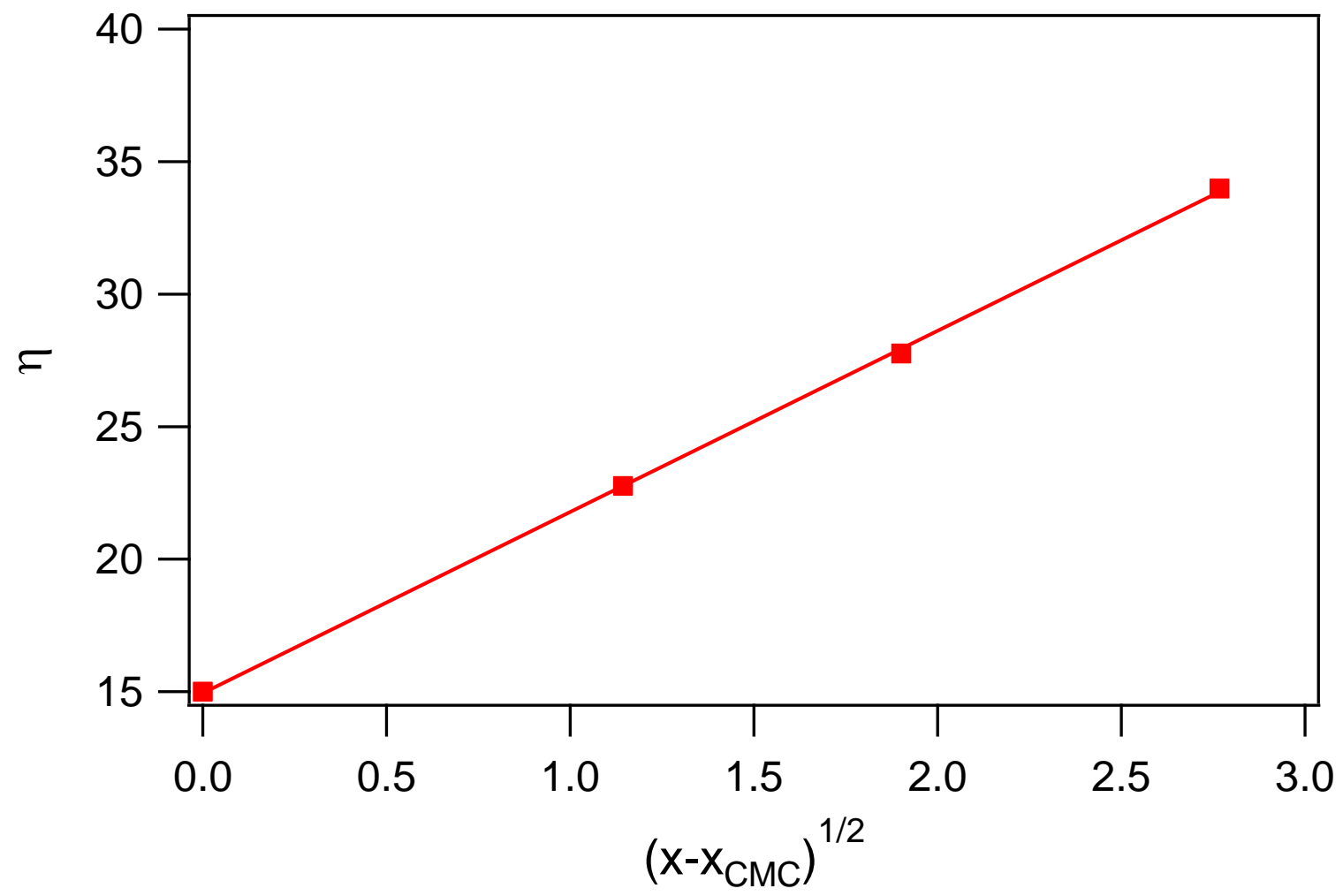

Figure 4. Ladder model fit for aggregation numbers of sodium bis(2-ethylhexyl) sulfosuccinate micelles in aqueous solutions. $\mathrm{X}$ is the mole fraction of the total surfactant present in solution and $X_{C M C}$ is the mole fraction of total surfactant present in solution at the critical micelle concentration. Markers in the figure represent the aggregation numbers extracted from the model fits to SANS data, and the solid line represents a linear fit to the aggregation number data $\eta=\eta_{0}+c_{1} \sqrt{X-X_{C M C}}$ where $\eta_{0}$ is the aggregation number for the micelles at the CMC. 


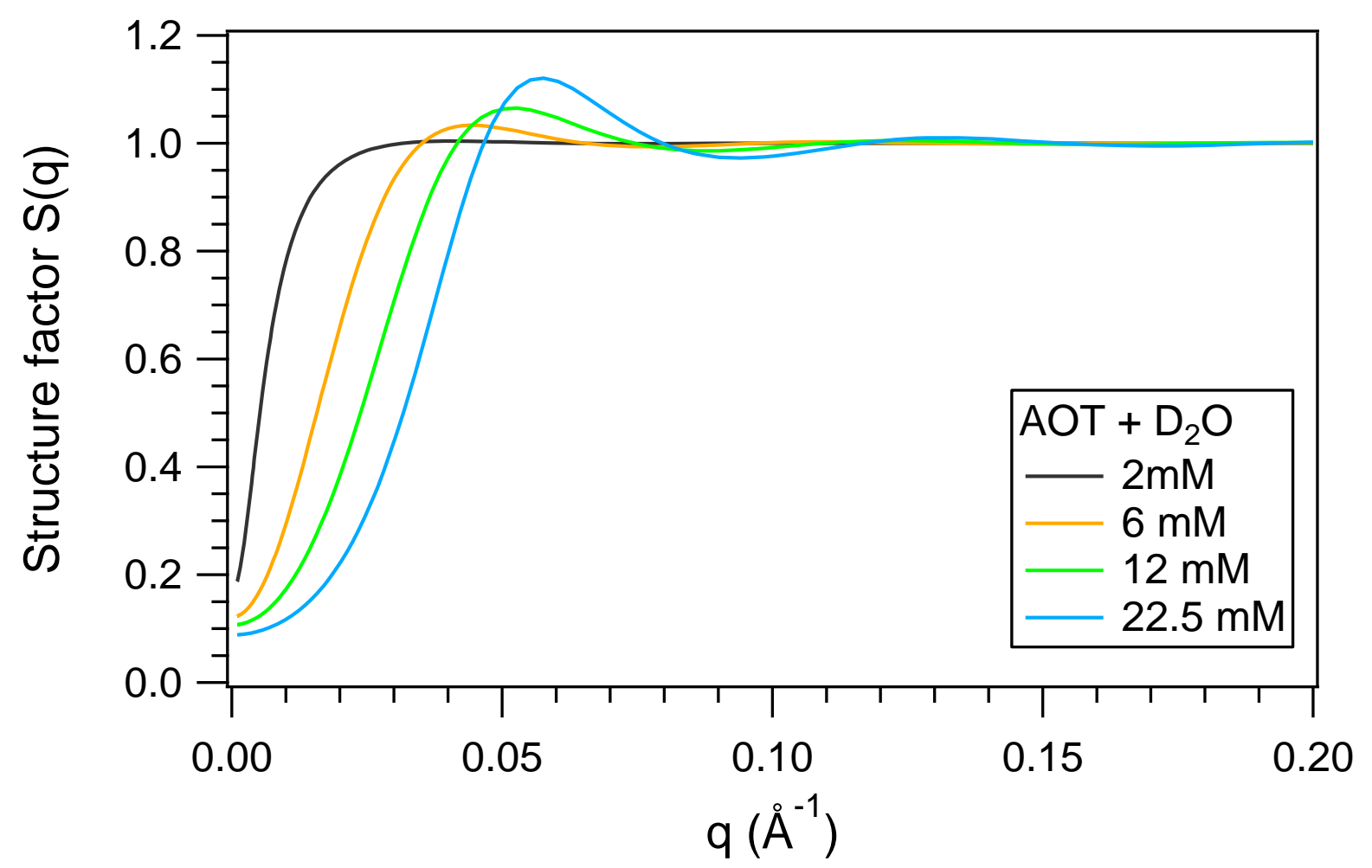

Figure 5. Structure factors extracted from SANS fits of scattering from sodium bis(2-ethylhexyl) sulfosuccinate micelles in $\mathrm{D}_{2} \mathrm{O}$ at different surfactant concentrations. 

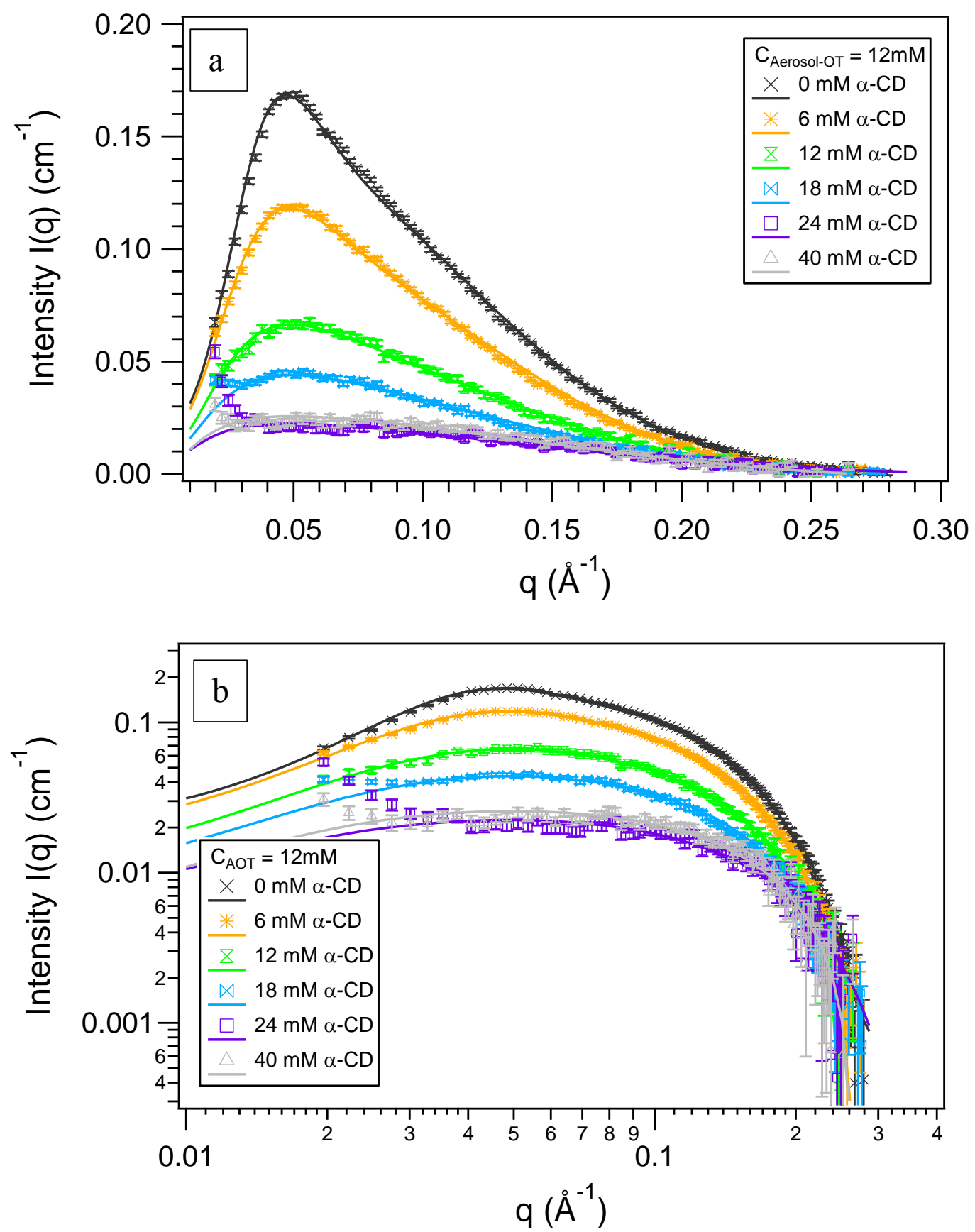

Figure 6. Effect of added $\alpha$-CD on SANS intensity profiles from $12 \mathrm{mM}$ AOT solutions in $\mathrm{D}_{2} \mathrm{O}$ in (a) linear plot, and (b) log-log plot. Markers represent experimental data and solid lines represent the corresponding fits using the core-shell oblate ellipsoid form factor with HansenHayter structure factor. 


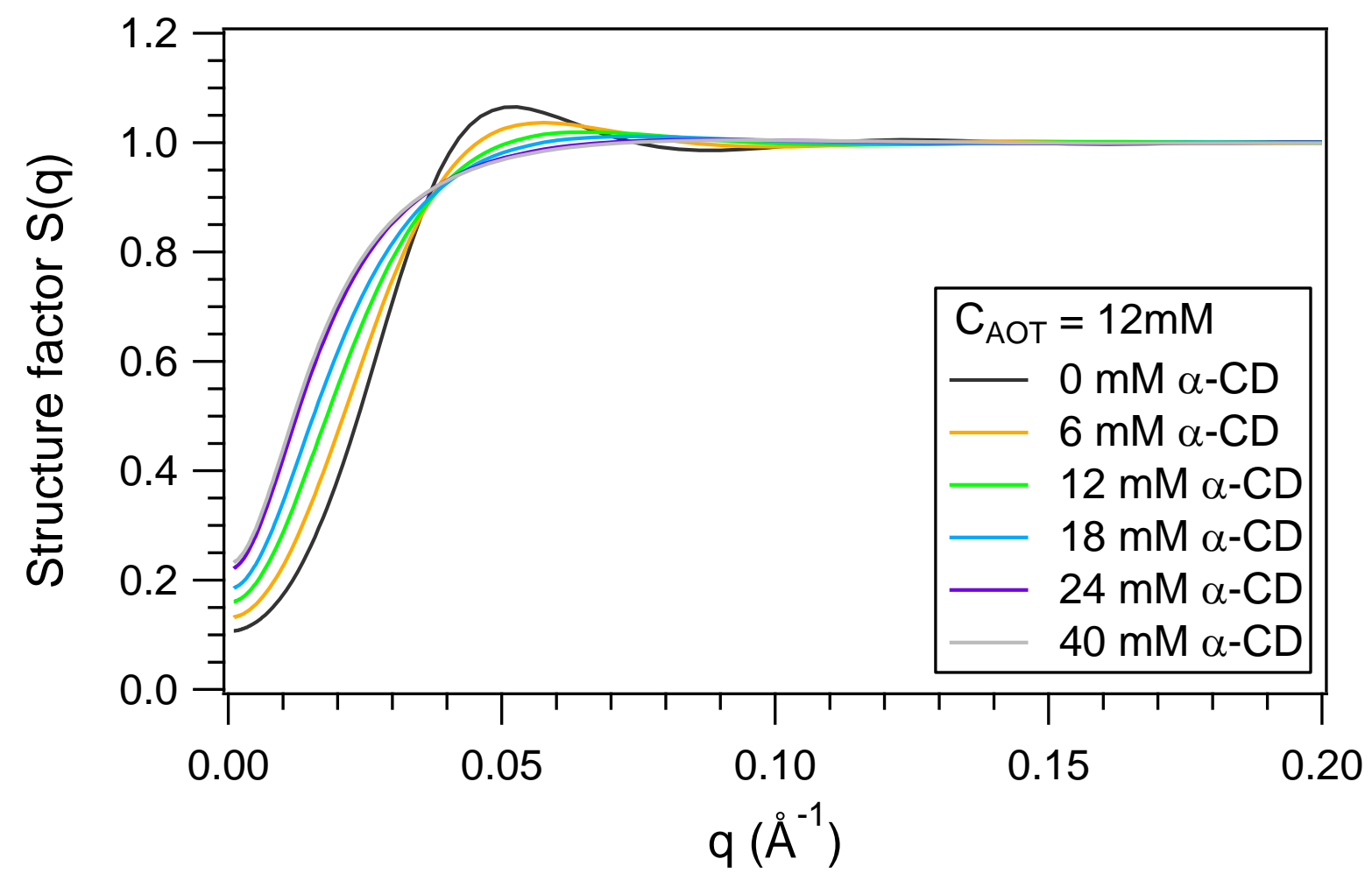

Figure 7. Structure factors extracted from SANS fits of scattering from AOT micelles in the presence of $\alpha-\mathrm{CD}$ at different concentrations (a fraction of $\mathrm{CD}$ may localize in micelle core and the remainder stay in the solution). 

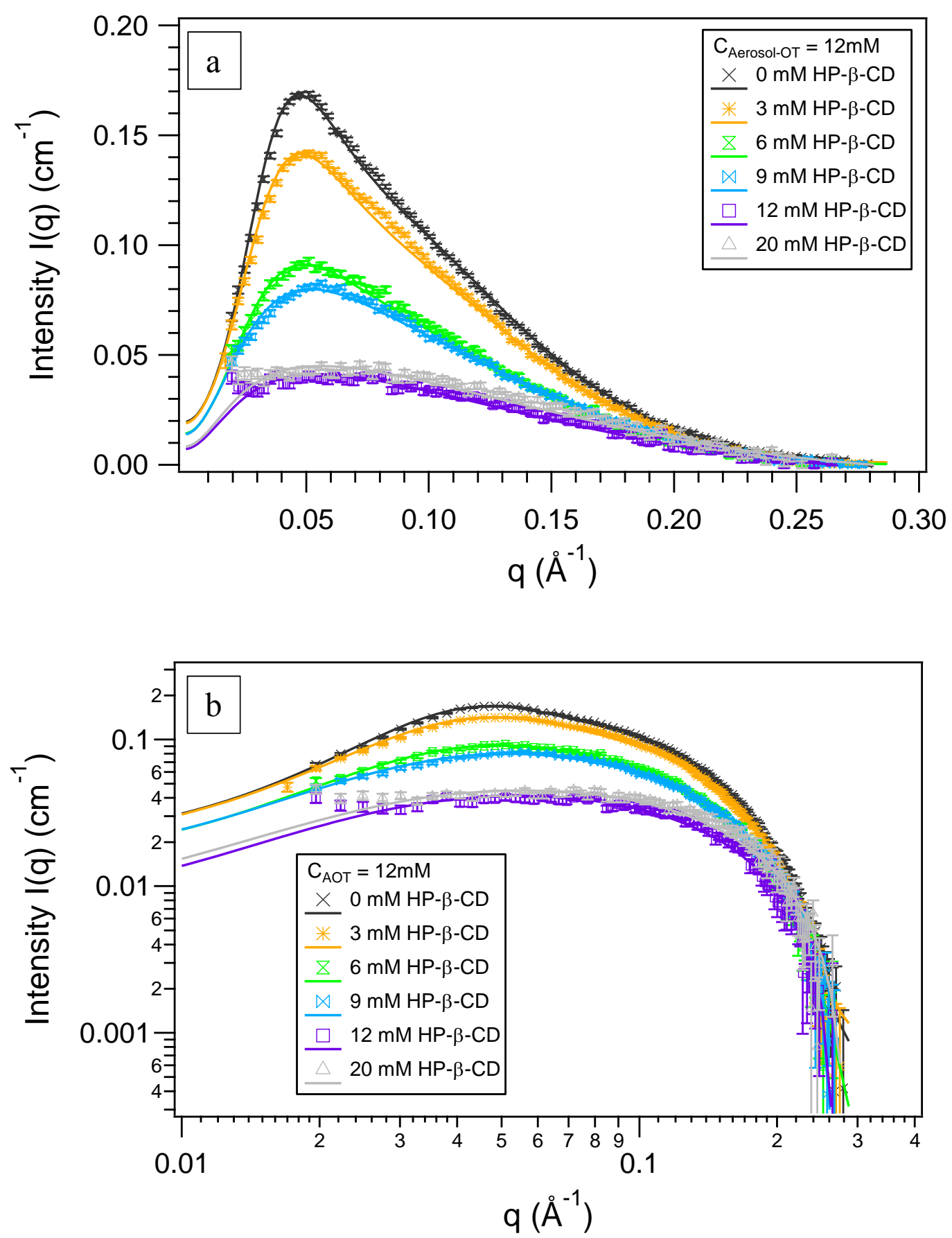

Figure 8: Effect of added HP $\beta-C D$ on SANS intensity profiles from $12 \mathrm{mM}$ AOT solutions in $\mathrm{D}_{2} \mathrm{O}$ in (a) linear plot, and (b) log-log plot. Markers represent experimental data and solid lines represent the corresponding fits using the core-shell oblate ellipsoid form factor and HansenHayter structure factor. 

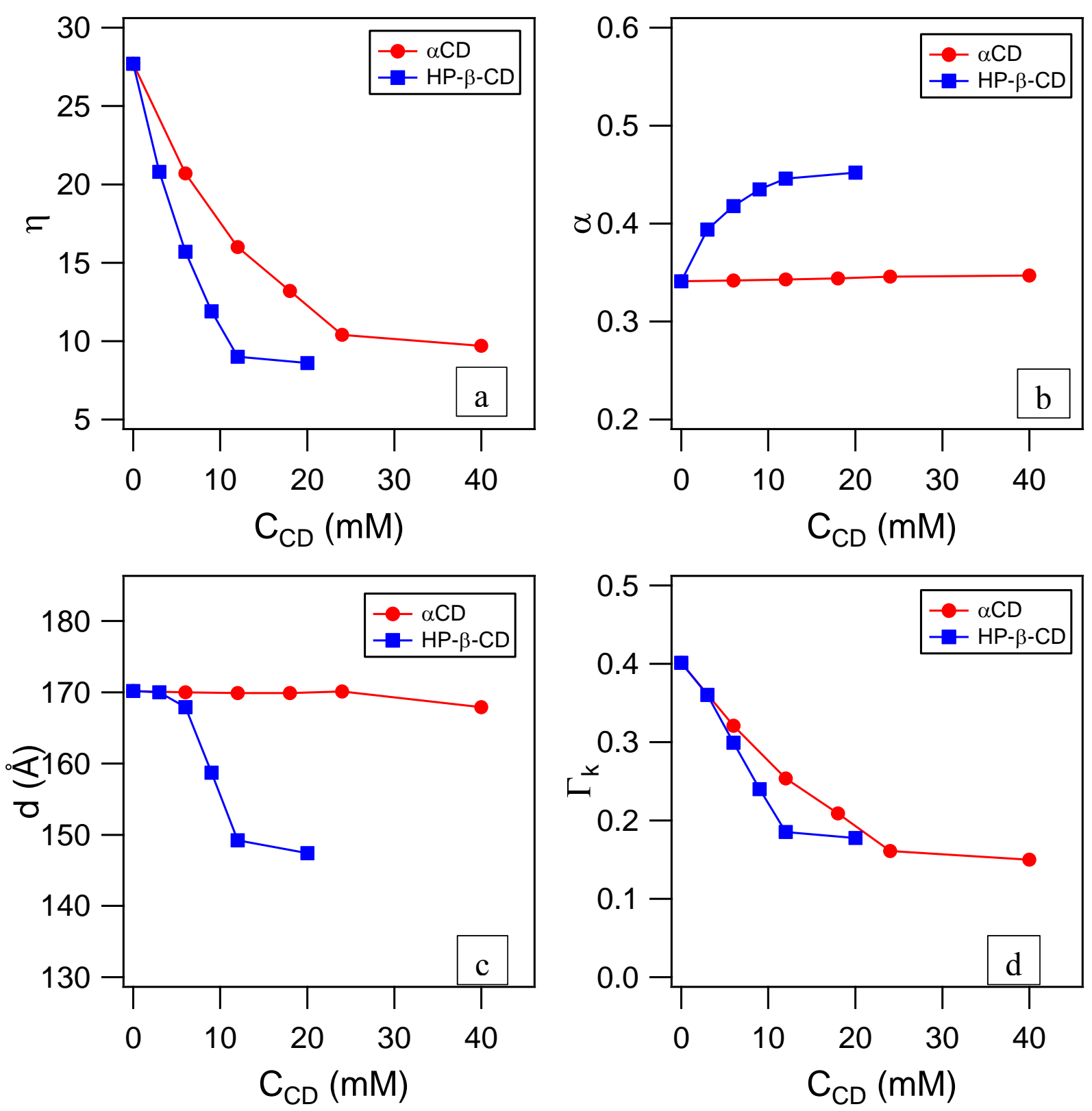

Figure 9: Effect of cyclodextrin type and concentration on (a) surfactant aggregation number $\eta$, (b) degree of dissociation of micelles $\alpha$, (c) inter-micelle distance $d$ and (d) electrostatic coupling constant $\Gamma_{k}$ for micelles formed by $12 \mathrm{mM}$ AOT in water. 


\section{List of symbols}

$a=$ Major radius of the micelle core $(\AA)$

$b=$ Minor radius of the micelle core $(\AA)$

$b_{i}=$ Scattering lengths of species $i(\AA)$

$C_{C D}=$ Concentration of cyclodextrin $(\mathrm{CD})(\mathrm{mM})$

$C M C_{S A N S}=$ Critical Micelle Concentration $(\mathrm{CMC})(\mathrm{mM})$

$C_{A O T}=$ Concentration of Aerosol-OT (AOT) $(\mathrm{mM})$

$d=$ Inter-micelle distance $(\AA)$

$D_{C D}=$ Outer diameter of $\mathrm{CD} \operatorname{ring}\left(\AA^{3}\right)$

$e=$ Charge of an electron (1.60217646 E-19 coulombs)

$k=$ Number of $\mathrm{CD}$ molecules in one micelle (Aggregation number of $\mathrm{CD}$ )

$K_{B}=$ Boltzmann constant $\left(1.3806503 \mathrm{E}-23 \mathrm{~m}^{2} \mathrm{~kg} \mathrm{~s}^{-2} \mathrm{~K}^{-1}\right)$

$M W_{D_{2} O}=$ Molecular weight of $\mathrm{D}_{2} \mathrm{O}\left(\mathrm{g} \cdot \mathrm{mole}^{-1}\right)$

$N_{A v}=$ Avogadro Number (6.023 E23)

$N_{H}=$ Hydration number (Number of water molecule associated with each surfactant)

$N_{P}=$ Micelle number density (Number of micelles $/ \AA^{3}$ )

$q=$ Scattering wave vector $\left(\AA^{-1}\right)$

$T=$ Absolute temperature $\left({ }^{\circ} \mathrm{K}\right)$

$V_{C}=$ Volume of micelle core $\left(\AA^{3}\right)$

$V_{C D}=$ Volume of a single $\mathrm{CD}$ molecule $\left(\AA^{3}\right)$

$V_{\mathrm{OSO}_{2}^{-}}, V_{\mathrm{Na}^{+}}, V_{\mathrm{D}_{2} \mathrm{O}}=$ Volumes of $\mathrm{OSO}_{2}^{-}, \mathrm{Na}^{+}$ions, and $\mathrm{D}_{2} \mathrm{O}$ molecules $\left(\AA^{3}\right)$

$V_{\text {Shell }}=$ Volume of shell in a micelle $\left(\AA^{3}\right)$

$V_{T O T}=$ Total volume of micelle $($ core + shell $)\left(\AA^{3}\right)$

$V t_{A O T}=$ Volume of AOT hydrocarbon tail $\left(\AA^{3}\right)$

$Z$ = Total charge on a micelle 


\section{Greek symbols:}

$\alpha=$ Fractional charge on a micelle

$\varepsilon=$ Dielectric constant of the solvent $\left(78.25\right.$ for $\left.\mathrm{D}_{2} \mathrm{O}\right)$

$\varepsilon_{0}=$ Permittivity of the free space

$\varphi=$ Volume fraction of micelles

$\Gamma=$ Fraction of CDs going in the micelles

$\Gamma_{k}=$ Dimensionless electrostatic interactions at inter-micelle distances (coupling constant)

$\kappa=$ Inverse screening length of Debye-Huckle theory $\left(\AA^{-1}\right)$

$\mu=$ Angle at which the major axis of a prolate is inclined to the scattering vector $q$ (radian)

$\eta=$ Aggregation number (number of surfactant molecules per micelle)

$\rho_{C}, \rho_{S h}, \rho_{S}=$ Scattering length density of micelle core, micelle shell, and solvent $\left(\AA^{-2}\right)$

$\rho_{D_{2} \mathrm{O}}^{\text {mass }}=$ Bulk density of $\mathrm{D}_{2} \mathrm{O}\left(\mathrm{g} \cdot \mathrm{cm}^{-3}\right)(1.1$ at room temperature $)$

$\sigma_{0}=$ Equivalent spherical $(\mathrm{OCM})$ diameter a micelle $(\AA)$

$\psi=$ Surface potential on a micelle $(\mathrm{mV})$ 